\title{
Conformal Blocks of Minimal Models on a Riemann Surface
}

\author{
G. Felder ${ }^{1}$ and R. Silvotti ${ }^{2, \star}$ \\ ${ }^{1}$ Institut für theoretische Physik, ETH-Hönggerberg, CH-8093 Zürich, Switzerland \\ 2 Mathematical Sciences Research Institute, Berkeley, CA 94720, USA
}

Received March 5, 1991

\begin{abstract}
We give explicit integral representations for conformal blocks of minimal models on arbitrary compact Riemann surfaces.
\end{abstract}

\section{Introduction}

Rational conformal field theory on the Riemann sphere $S$ can be formulated in terms of a pair $\mathscr{L} \oplus \mathscr{L}$ of identical Virasoro algebras $\mathscr{L}$ with assigned rational central charge $c$. If $L(h, c)$ denotes the irreducible highest weight $\mathscr{L}$-module of highest weight $h$, the Hilbert space of the theory decomposes as $\bigoplus_{h, h^{\prime}} L(h, c) \otimes L\left(h^{\prime}, c\right)$, with $h$ and $h^{\prime}$ ranging in some finite set of rational values. Correlation functions of local fields at point $P_{1}, \ldots, P_{n}$ on $S$ admit an analogous decomposition into the so-called left (respectively right) conformal blocks, which depend holomorphically (respectively antiholomorphically) on the local coordinates $z\left(P_{i}\right)$ defined about the $P_{i}$.

Under certain consistency requirements, the theory can be generalized to Riemann surfaces $\Sigma$ of positive genus. The main argument for this lies in a formalization of the surgery operations ("sewing") through which $\Sigma$ can be obtained from a set of three-punctured spheres. General formulations of conformal field theory on Riemann surfaces have been outlined by Segal and by Gawedzki. They can be roughly summarized as follows: The holomorphic part of a conformal field theory is specified by assignments $\Sigma \mapsto B(\Sigma)$ of objects $B$ to Riemann surfaces. If $\Sigma$ has $m+n$ punctures at points $P_{i}$, the $B(\Sigma): \bigotimes_{1 \leqq i \leqq m} L\left(h_{i}, c\right) \rightarrow \bigotimes_{m+1 \leqq i \leqq m+n} L\left(h_{i}, c\right)$ are trace class operators depending holomorphically on $z\left(P_{i}\right)$ and having specified properties under conformal diffeomorphisms. Moreover, the assignment $B$ from

* On leave from Columbia University, Department of Mathematics, New York, NY 10027, USA Supported by NSF under grant DMS- 8505550 
Riemann surfaces and a set of surgery operations to operators and trace operations has functorial properties ("sewing axioms"); the "functor" $B$ is therefore specified by the assignments of trilinear operators $B(X)$ to three-punctured spheres $X$.

In this paper, we present a calculation of $B$ for the minimal models $M_{p, p^{\prime}}$ of Belavin, Polyakov and Zamolodchikov. These are the conformal field theories constructed upon the irreducible highest weight modules of $\mathscr{L}$ with central charge $c=1-6\left(p-p^{\prime}\right)^{2} / p p^{\prime}$ parametrized by two positive and relatively prime integers $p$ and $p^{\prime}$.

In the first step (see Sect. 2), we use the fact that the relevant irreducible modules $L(h, c)$ are isomorphic to the cohomologies of certain complexes $\mathscr{F}$ of Fock spaces ("BRST cohomology"). This identification allows us to make use of free field techniques.

In a second step we construct the trilinear operators $B(X)$. These are the dual objects of a complex trilinear form $C$ (the "three-point vertex") defined on the product of three irreducible modules and associated with the sphere with three punctures. $C$ is, in principle, determined up to a normalization constant by the conformal Ward identities. In Sect. 3, $C$ will be constructed as the trilinear form induced on BRST cohomology by a trilinear form $\mathscr{V}$ defined on the product of Fock complexes. Such $\mathscr{V}$ can be given an explicit representation in terms of integrals of conformal blocks of free fields along suitable twisted cycles. The construction of $C$ is then reduced to the calculation of certain twisted homology groups. Although the calculation is not completely rigorous, owing to technical difficulties related to support conditions in locally finite homology, it gives a very consistent picture. In particular, the fusion rules appear, when one considers the space of homology cycles giving rise to three-point vertices which are well-defined on BRST cohomology, modulo the space of homology cycles giving rise to threepoint vertices which vanish on BRST cohomology.

In a third step we construct integral representations for general conformal blocks by the sewing procedure: the Riemann surface is represented as the result of sewing three-holed spheres, and conformal blocks are labeled by all possible assignments of triples of highest weight modules to the three-holed spheres, such that the modules associated to the holes to be sewn together are one the dual of the other. Conformal blocks are the result of the contraction of the corresponding trilinear forms. It is believed (although no complete mathematical proof exists to our knowledge) that this construction of the vector space of conformal blocks is independent of the choice of the sewing pattern, provided one has two consistency conditions which can be checked in genus zero and one, and have indeed been checked for these models.

Our results for general conformal blocks are complete for minimal models $M_{p p^{\prime}}$ with $p^{\prime}=2$ or $p=2$, i.e. the models where only one type of screening charge is present. In the general case the integral expressions for conformal blocks have an integrable singularity at the points in the integration domain where screening charges of the two different types coincide. At these points one has in general a local violation of Ward identities. We conjecture that a suitable regularization procedure at those points should give conformal blocks obeying the Ward identities. We have checked that there is such a regularization for three-point vertices in which only one screening charge of each type appears, but do not have a general proof. 
Techniques analogous to the ones employed in this paper have recently been used in [DaJMM, FeiSV, L, Ma, ScV] to compute solutions to the KnizhnikZamolodchikov equation.

\section{Representations of the Kac Discrete Series}

We consider the Virasoro algebra $\mathscr{L}$ over $\mathbb{C}$, with generators $e_{n}(n \in \mathbb{Z})$ and commutation relations

$$
\left[e_{m}, e_{n}\right]=(m-n) e_{m+n}+\frac{c}{12}\left(m^{3}-m\right) \delta_{m,-n},
$$

where the central charge $c=c\left(\beta_{0}\right)=1-12 \beta_{0}^{2}$ is a $c$-number parametrized by $\beta_{0} \in \mathbb{C}$.

Let us recall a few well-known properties of the representations of $\mathscr{L}$. The Verma module $V(h, c)$ of weight $h$ is the left $\mathscr{L}$-module provided with a highest weight vector $w$, with properties: $e_{0} w(h)=h w(h) ; e_{k} w(h)=0, k>0$. It is spanned by the vectors $e_{-k_{1}} \cdots e_{-k_{r}} w(h)\left(1 \leqq k_{1} \leqq \cdots \leqq k_{r}, r=0,1, \ldots\right)$. In particular, we will consider the set of weights

$$
h_{n n^{\prime}}=\frac{1}{8}\left(n^{2}-1\right) \beta_{+}^{2}-\frac{1}{2}\left(n n^{\prime}-1\right)+\frac{1}{8}\left(n^{\prime 2}-1\right) \beta_{-}^{2},
$$

where $n, n^{\prime} \in \mathbb{Z}$ and $\beta_{ \pm}=\beta_{0} \pm \sqrt{2+\beta_{0}^{2}}$. The module $V\left(h_{n n^{\prime}}, c\right)$ with weight in (2.1) is irreducible if $n n^{\prime} \leqq 0$. If $n n^{\prime}>0$, then $V\left(h_{n n^{\prime}}, c\right)$ is reducible and the irreducible highest weight module $L\left(h_{n n^{\prime}}, c\right)$ with weight $h_{n n^{\prime}}$ is realized as the quotient of $V\left(h_{n n^{\prime}}, c\right)$ by its maximal proper submodule.

A resolution of the irreducible modules $L\left(h_{n n^{\prime}}, c\right)$ in terms of Verma modules was given by Feigin and Fuchs [FeiFu]. If $\beta_{+}^{2} / 2=p^{\prime} / p$ is a positive rational number, the central charge $c=1-6\left(p-p^{\prime}\right)^{2} / p p^{\prime}$ is rational and lower than one. The corresponding irreducible modules $L\left(h_{n n^{\prime}}, c\right)$ form the so-called Kac discrete series. The associated conformal field theory is the one of minimal models [BPZ].

Let $\mathscr{A}$ be the Heisenberg algebra over $\mathbb{C}$, with generators $a_{n}(n \in \mathbb{Z})$ and relations $\left[a_{m}, a_{n}\right]=m \delta_{m,-n}$. The generators of $\mathscr{L}$ can be obtained by a deformation of the Sugawara construction:

$$
\begin{aligned}
& L_{n}=\frac{1}{2} \sum_{k \in \mathbb{Z}} a_{n-k} a_{k}-\beta_{0}(n+1) a_{n}, \quad n \neq 0, \\
& L_{0}=\sum_{k=1}^{\infty} a_{-k} a_{k}+\frac{1}{2} a_{0}^{2}-\beta_{0} a_{0} .
\end{aligned}
$$

The Fock space $F\left(\beta, \beta_{0}\right)$ of charge $\beta$ is the left $\mathscr{L}$-module built upon a highest weight vector $v(\beta)$ (having properties: $a_{0} v(\beta)=\beta v(\beta) ; a_{k} v(\beta)=0, k>0$ ) with weight $h(\beta)=\frac{1}{2} \beta^{2}-\beta_{0} \beta$. We have the canonical homomorphism $\phi_{\beta}: V(h(\beta), c) \rightarrow F\left(\beta, \beta_{0}\right)$ between $\mathscr{L}$-modules defined by $\phi_{\beta} w(h(\beta))=v(\beta)$ and $\phi_{\beta} e_{n}=L_{n} \phi_{\beta}$ for $n \in \mathbb{Z}$. Note that $\phi_{\beta}$ is an isomorphism only if $V(h(\beta), c)$ is irreducible. The relation between Verma and Fock modules was analyzed in [Fel 1] in the case $V(h(\beta), c)$ is reducible. The result says, in words, that the irreducible modules $L\left(h_{n n^{\prime}}, c\right)$ with weights in (2.1) are realized as cohomologies of suitably defined complexes of Fock spaces 
$F\left(\beta_{n n^{\prime}}, \beta_{0}\right)$ with charges

$$
\beta_{n n^{\prime}}=\frac{1}{2}(1-n) \beta_{+}+\frac{1}{2}\left(1-n^{\prime}\right) \beta_{-}, \quad n, n^{\prime} \in \mathbb{Z},
$$

and highest weights $h\left(\beta_{n n^{\prime}}\right)=h_{n n^{\prime}}$. The construction of such complexes (see Theorem 1) requires some preliminaries.

Let $b_{0}$ be an extra-element appended to the Heisenberg algebra, with $\left[b_{0}, a_{n}\right]=i \delta_{0, n}$. Thus $e^{i a b_{0}}$ maps $F\left(\beta, \beta_{0}\right)$ to $F\left(\beta+\alpha, \beta_{0}\right)$. For every $\alpha, \beta \in \mathbb{C}$, the vertex operator $V_{\alpha}(z)$ is an element of $\operatorname{Hom}_{\mathbb{C}}\left(F\left(\beta, \beta_{0}\right), F\left(\beta+\alpha, \beta_{0}\right)\right)$ defined as the formal power series

$$
V_{\alpha}(z)=e^{i \alpha b_{0}} z^{\alpha a_{0}} \exp \left(\alpha \sum_{n=1}^{\infty} \frac{a_{-n}}{n} z^{n}\right) \exp \left(-\alpha \sum_{n=1}^{\infty} \frac{a_{n}}{n} z^{-n}\right) .
$$

We introduce the $\mathscr{L}$-module $F\left(\beta, \beta_{0}\right)^{\vee}$ dual to $F\left(\beta, \beta_{0}\right)$. It is the space of covectors $\omega$ defined by the requirement that $\left\langle L_{n} \omega, \xi\right\rangle=\left\langle\omega, L_{-n} \xi\right\rangle$ for all $\xi \in F(\beta)$, with the normalization convention $\left\langle v(\beta)^{\vee}, v(\beta)\right\rangle=1$ between highest weight vectors $v(\beta)^{\vee}$ and $v(\beta)$. It follows that $v(\beta)^{\vee}$ has the same weight $h(\beta)$ as $v(\beta)$. It can be easily shown that $F(\beta, \beta)^{\vee}$ is also a Fock space and that it is isomorphic to $F\left(2 \beta_{0}-\beta, \beta_{0}\right)$. With this notion of dual Fock modules, the vertex operator $V_{\alpha}(z)$ may also be viewed as an infinite matrix with elements $\left\langle\omega, V_{\alpha}(z) \xi\right\rangle$, where the vectors $\xi=a_{-k_{1}} \cdots$ $a_{-k_{r}} v(\beta)\left(1 \leqq k_{1} \leqq \cdots \leqq k_{r} ; r=0,1, \ldots\right)$ are the elements of a basis for $F\left(\beta, \beta_{0}\right)$ and the covectors $\omega \in F\left(\beta+\alpha, \beta_{0}\right)^{\vee}$ are the dual of the elements of an analogously chosen basis for $F\left(\beta+\alpha, \beta_{0}\right)$. These matrix elements are multivalued analytic functions of $z \in \mathbb{C}-\{0\}$.

Similarly, the product of vertex operators $V_{\alpha_{i}}\left(z_{i}\right)(i=1, \ldots, n)$ has well defined matrix elements between bases of $F\left(\beta, \beta_{0}\right)$ and $F\left(\beta+\sum_{i} \alpha_{i}, \beta_{0}\right)^{\vee}$. We compute its value around a point $P_{0} \in Z(n)=(\mathbb{C}-\{0\})^{n}-\bigcup_{i<j}\left\{z_{i}=z_{j}\right\}$, where the arguments are ordered as $\left|z_{1}\right|>\cdots>\left|z_{n}\right|$ :

$$
\begin{aligned}
V_{\alpha_{1}}\left(z_{1}\right) \cdots V_{\alpha_{n}}\left(z_{n}\right)= & \prod_{i<j}\left(z_{i}-z_{j}\right)^{\alpha_{i} \alpha_{j}} \exp \left(i \sum_{i} \alpha_{i} b_{0}\right) \prod_{i} z_{i}^{\alpha_{i} a_{0}} \\
& \cdot \exp \left(\sum_{i} \sum_{n=1}^{\infty} \alpha_{i} \frac{a_{-n}}{n} z_{i}^{n}\right) \exp \left(-\sum_{i} \sum_{n=1}^{\infty} \alpha_{i} \frac{a_{n}}{n} z_{i}^{-n}\right) .
\end{aligned}
$$

At every other point $P \in Z(n)$ its value is then determined, for each homotopy class

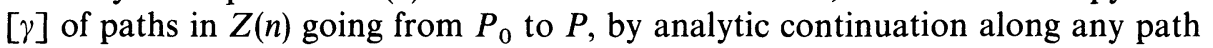
$\gamma \in[\gamma]$.

We define the weight $h(\alpha)$ of the vertex operator $V_{\alpha}(z)$ by the commutation relations

$$
\left[L_{k}, V_{\alpha}(z)\right]=\left(z^{k+1} \frac{d}{d z}+h(\alpha)(k+1) z^{k}\right) V_{\alpha}(z)
$$

We have $h(\alpha)=\frac{1}{2} \alpha^{2}-\beta_{0} \alpha$. Vertex operators satisfying the above relations are said primary. In particular, $V_{\beta_{+}}(z)$ and $V_{\beta_{-}}(z)$ (screening operators) have weight $h\left(\beta_{ \pm}\right)=1$, and their commutators with $L_{k}$ are equal to the derivatives of $z^{k+1} V_{\beta_{ \pm}}(z)$. More in general, the (matrix elements of the) operators mapping $F\left(\beta, \beta_{0}\right)$ to $F\left(\beta+m \beta_{+}, \beta_{0}\right)$, defined as

$$
\omega_{m}=V_{\beta+}\left(z_{1}\right) \cdots V_{\beta+}\left(z_{m}\right) d z_{1} \wedge \cdots \wedge d z_{m},
$$


are multivalued holomorphic $m$-forms on the quotient space $Z(m) / S(m)$, with $S(m)$ the permutation group of $m$ elements, and satisfy $\left[L_{k}, \omega_{m}\right]=d \eta$, where $\eta$ is a holomorphic $(m-1)$-form having the same monodromy as $\omega_{m}$. Thus

$$
Q_{m}=\int_{d(m)} \omega_{m}
$$

commutes with the Virasoro algebra if $d(m)$ has no boundary. As we will see in a more general case in Sect. 3, $d(m)$ is an $m$-cycle for the homology of $Z(m) / S(m)$ with coefficients in a local system associated with the monodromy of $\omega_{m}$. Since in this case the homology is one-dimensional, the integral $Q_{m}$ is determined up to a multiplicative factor.

Henceforth $\beta_{+}^{2} / 2=\left(\beta_{-}^{2} / 2\right)^{-1}=p^{\prime} / p$, with $p$ and $p^{\prime}$ two positive integers chosen to have no common divisor. In this case note that the charges (2.2) are not all distinct; we have in fact $\beta_{n n^{\prime}}=\beta_{n+p, n^{\prime}+p^{\prime}}$ for all $n^{\prime}, n \in \mathbb{Z}$. It is in virtue of these relations that one can define the sequence at point (i) of the following theorem.

Theorem 1 [Fel 1]. If $\beta_{+}^{2} / 2=p^{\prime} / p$, let $\left(m, m^{\prime}\right)$ be a pair in the Kac table $D=\left\{\left(m, m^{\prime}\right)\right.$

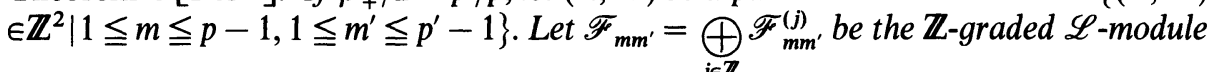
defined by $\mathscr{F}_{m m^{\prime}}^{(j)}=F\left(\beta_{m[j], m^{\prime}[j]}, \beta_{0}\right)$ with $\left(m[j], m^{j}[j]\right)=\left(m, m^{\prime}+j p^{\prime}\right)$ for $j$ even and $\left(m[j], m^{\prime}[j]\right)=\left(p-m, m^{\prime}+j p^{\prime}\right)$ for $j$ odd. Then:

(i) There exists a sequence of homomorphisms

$$
\cdots \stackrel{Q^{(-2)}}{\longrightarrow} \mathscr{F}_{m m^{\prime}}^{(-1)} \stackrel{Q^{(-1)}}{\longrightarrow} \mathscr{F}_{m m^{\prime}}^{(0)} \stackrel{Q^{(0)}}{\longrightarrow} \mathscr{F}_{m m^{\prime}}^{(1)} \stackrel{Q^{(1)}}{\longrightarrow \cdots}
$$

between Virasoro modules defined by $Q^{(j)} \xi=Q_{m} \xi$ for $j$ even and $Q^{(j)} \xi=Q_{p-m} \xi$ for jodd.

(ii) Let the cycle defining $Q_{m}$ be $d(m)=\prod_{l=1}^{m} \frac{e^{i \pi \beta_{+}^{2} l}-1}{e^{i \pi \beta_{+}^{2}}-1} d_{m}$, where $d_{m}=\left\{\left(z_{1}\left(\theta_{1}\right), \ldots\right.\right.$, $\left.\left.z_{m}\left(\theta_{m}\right)\right) \in T^{m} \mid 0 \leqq \theta_{1}<\cdots<\theta_{m} \leqq \pi\right\}$ and $T$ is a circle centered at $z=0$ parametrized by $\theta \in[0, \pi]$. Then $Q^{(j)} Q^{(j-1)}=0$ for all $j \in \mathbb{Z}$ and $\mathscr{F}_{m m^{\prime}}$ is a complex.

(iii) The cohomology groups $H^{j}\left(\mathscr{F}_{m m^{\prime}}\right)=\operatorname{Ker} Q^{(j)} / \operatorname{Im} Q^{(j-1)}$ vanish if $j \neq 0$ and $H^{0}\left(\mathscr{F}_{m m^{\prime}}\right)$ is isomorphic to the irreducible highest weight module $L\left(h_{m m^{\prime}}, c\right)$. (The isomorphism $\phi_{m m^{\prime}}$ is the canonical one mapping highest weight vector to highest weight vector.)

Note that the normalization of the cycle $d(m)$ has been chosen such to give a coboundary operator, i.e. such that $Q_{m} Q_{p-m}=0=Q_{p-m} Q_{m}$. One usually refers to $Q$ as to the BRST operator.

Note that, for $\beta_{+}^{2} / 2=p^{\prime} / p, 2 \beta_{0}-\beta_{m m^{\prime}}=\beta_{p-m, p^{\prime}-m^{\prime}}$. Hence, the complex $\mathscr{F}_{m m^{\prime}}{ }^{\vee}=\bigoplus_{j \in \mathbb{Z}} \mathscr{F}_{m m^{\prime}}^{(j) \vee}$ dual to $\mathscr{F}_{m m^{\prime}}$ is specified by the isomorphisms $\mathscr{F}_{m m^{\prime}}^{(j) \vee} \cong_{p-m, p^{\prime}-m^{\prime}}$ for $j \in \mathbb{Z}$ and $\left(m, m^{\prime}\right) \in D$.

\section{The Three-Point Vertex and Twisted Homology of Braid Spaces}

3.1. In this section we give the details of the construction of the three-points vertex of minimal models introduced in [FelS 2]. This object generalizes the intertwining operators introduced by Tsuchiya and Kanie [TK]. 
A pair of integers e.g. $\left(n, n^{\prime}\right)$, will be denoted by the corresponding capital letter, i.e. $\left(n, n^{\prime}\right)=N$. So also $\left(p, p^{\prime}\right)=P$; and $(1,1)$ will be denoted by 1 .

We will always identify the irreducible module $L\left(h_{M}, c\right)$ with index $M$ in the Kac table with the cohomology module $H^{*}\left(\mathscr{F}_{M}\right)$ of Theorem 1. Three-points conformal blocks of minimal models on the Riemann sphere $S$ depend on a triple $\mathrm{O}_{1}, \mathrm{O}_{2}, \mathrm{O}_{3}$ of distinct points on $\mathrm{S}$, and a choice of local coordinates vanishing at $O_{i}$. They are constructed as complex trilinear forms $C_{M_{1} M_{2} M_{3}}$ on the product $L\left(h_{M_{1}}, c\right) \times L\left(h_{M_{2}}, c\right) \times L\left(h_{M_{3}}, c\right)$ of irreducible modules with indices $M_{i}$ in the Kac table $\mathrm{D}$. And are determined, up to a normalization constant, by the conformal Ward identities [BPZ], which express a covariance property under the Lie algebra of meromorphic vector fields on $S$ holomorphic on $S-\left\{O_{1}, O_{2}, O_{3}\right\}$. If we fix two of the points, e.g. $O_{1}=\infty, O_{3}=0$, the operators obtained from $C_{M_{1} M_{2} M_{3}}$ by duality form the set of minimal models' chiral fields.

In the following paragraph we introduce trilinear forms $\mathscr{V}_{M_{1} M_{2} M_{3}}$ defined on the Fock complexes $\mathscr{F}_{M_{i}}$ of Theorem 1 and satisfying the Ward identities of $C_{M_{1} M_{2} M_{3}}$. By Theorem 2, these induce trilinear forms $\mathscr{V}_{M_{1} M_{2} M_{3}}^{*}$ on cohomologies $H^{*}\left(\mathscr{F}_{M_{i}}\right)$ which are identified with three-points conformal blocks. Such identification will be discussed below Theorem 2. Here it is important to note that, because $h_{M_{i}}=h_{P-M_{i}}$, there are canonical module isomorphisms $\psi_{i}^{*}: H^{*}\left(\mathscr{F}_{M_{i}}\right) \rightarrow H^{*}\left(\mathscr{F}_{P-M_{i}}\right)$. Thus all composites $\left(\mathscr{V}^{*} \cdot \psi_{1}^{*}\right)_{M_{1} M_{2} M_{3}},\left(\mathscr{V}^{*} \cdot \psi_{2}^{*}\right)_{M_{1} M_{2} M_{3}},\left(\mathscr{V}^{*} \cdot \psi_{3}^{*}\right)_{M_{1} M_{2} M_{3}},\left(\mathscr{V}^{*} \cdot \psi_{1}^{*} \cdot \psi_{2}^{*}\right)_{M_{1} M_{2} M_{3}}$ are trilinear forms on cohomologies $H^{*}\left(\mathscr{F}_{M_{i}}\right)$ satisfying the same Ward identities, and can be equivalently identified with $C_{M_{1} M_{2} M_{3}}$.

3.2. Let us suppose that $O_{i} \neq \infty$ and that the local coordinates around $O_{i}$ are $z-z\left(O_{i}\right)$, where $z$ is the standard coordinate on $\mathbb{C}=S-\infty$. Other choices are related to this one by conformal transformations.

Define descendant vertex operators $V(\xi, P)$ at $P \in \mathbb{C}$ with local coordinate $z-z(P)$, depending linearly on $\xi \in F\left(\alpha, \beta_{0}\right)$, as follows: if $\xi=\prod_{j} a_{-k_{j}} v(\alpha)$, then, in
terms of the $U(1)$-current $J(z)=\sum a_{n} z^{-n-1}$,

$$
V(\xi, P)=\prod \oint \frac{d z_{j}}{2 \pi i}\left(z_{j}-z(P)\right)^{k_{j}} J\left(z_{j}\right) V_{\alpha}(P)
$$

with integration over small circles encircling $P$. The definition is then extended by linearity to the whole Fock space. As for vertex operators, vacuum expectation values of products of descendant vertex operators have well defined meaning as analytic continuation of absolutely convergent series and integrals, and are manyvalued functions with the same monodromy as the corresponding product of primary vertex operators $V_{\alpha}(P)=V(v(\alpha), P)$.

Let $O_{1}, O_{2}$ and $O_{3}$ be three points on $S$. In the continuation of this section: The $M_{i}$ for $i=1,2,3$ are pairs in the Kac table $D=\left\{1 \leqq m \leqq p-1,1 \leqq m^{\prime} \leqq p^{\prime}-1\right\}$. For $j_{i} \in \mathbb{Z}(i=1,2,3)$ such that $\sum_{i} j_{i}=0$, the $M_{i}\left[j_{i}\right]=\left(m_{i}\left[j_{i}\right], m_{i}^{\prime}\left[j_{i}\right]\right)$ are the pairs $\left(m_{i}, m_{i}^{\prime}+j_{i} p^{\prime}\right)$ when $j_{i}$ is even and $\left(p-m_{i}, m_{i}^{\prime}+j_{i} p^{\prime}\right)$ when $j_{i}$ is odd.

The three-points vertex $\mathscr{V}_{M_{1} M_{2} M_{3}}\left(O_{1}, O_{2}, O_{3}\right): \mathscr{F}_{M_{1}} \times \mathscr{F}_{M_{2}} \times \mathscr{F}_{M_{3}} \rightarrow \mathbb{C}$ is a trilinear form of degree zero on Fock complexes. Its component on $\mathscr{F}_{M_{1}}^{\left(j_{1}\right)} \times \mathscr{F}_{M_{2}}^{\left(j_{2}\right)} \times$ $\mathscr{F}_{M_{3}}^{\left(j_{3}\right)} \ni\left(\xi_{1}, \xi_{2}, \xi_{3}\right)$ is defined by the integral

$$
\begin{aligned}
& \mathscr{V}_{M_{1}\left[j_{1}\right] M_{2}\left[j_{2}\right] M_{3}\left[j_{3}\right]}\left(O_{1}, O_{2}, O_{3} ; \xi_{1}, \xi_{2}, \xi_{3}\right) \\
& =\int_{c_{M_{1}\left[j_{1}\right] M_{2}\left[j_{2}\right] M_{3}\left[j_{3}\right]}} \omega_{M_{1}\left[j_{1}\right] M_{2}\left[j_{2}\right] M_{3}\left[j_{3}\right]}\left(O_{1}, O_{2}, O_{3} ; \xi_{1}, \xi_{2}, \xi_{3}\right) .
\end{aligned}
$$


The integrand is the matrix element of an operator mapping the vacuum Fock space $F\left(0, \beta_{0}\right)$ to the equivalent (in cohomology) Fock space $F\left(\beta_{P-1}, \beta_{0}\right)$,

$$
\begin{aligned}
\omega_{M_{1}\left[j_{1}\right] M_{2}\left[j_{2}\right] M_{3}\left[j_{3}\right]}\left(O_{1}, O_{2}, O_{3} ; \xi_{1}, \xi_{2}, \xi_{3}\right) \\
=\left\langle v\left(\beta_{P-1}\right)^{\vee}, V\left(\xi_{1}, O_{1}\right) V\left(\xi_{2}, O_{2}\right) V\left(\xi_{3}, O_{3}\right) \prod_{i=1}^{r[j]} V_{\beta+}\left(P_{i}\right) \prod_{i^{\prime}=1}^{r^{\prime}[j]} V_{\beta_{-}}\left(P_{i^{\prime}}^{\prime}\right) v(0)\right\rangle \\
\quad \cdot d z\left(P_{1}\right) \wedge \cdots \wedge d z\left(P_{1}^{\prime}\right) \wedge \cdots,
\end{aligned}
$$

if the numbers

$$
\begin{aligned}
r[j] & =\frac{1}{2}\left(\sum_{i} m_{i}\left[j_{i}\right]-p-1\right) \\
r^{\prime}[j] & =\frac{1}{2}\left(\sum_{i} m_{i}^{\prime}\left[j_{i}\right]-p^{\prime}-1\right)=\frac{1}{2}\left(\sum_{i} m_{i}^{\prime}-p^{\prime}-1\right),
\end{aligned}
$$

are non-negative integers. If this condition is not satisfied, the three-points vertex is defined to be zero.

The three-points vertex obeys the Ward identities if the domain of integration $c_{M_{1}\left[j_{1}\right] M_{2}\left[j_{2}\right] M_{3}\left[j_{3}\right]}$ is a cycle dual to the cohomology of the multivalued forms (3.2). The problem of determining this cycle is considered in the following paragraphs.

3.3. Here $p^{\prime}$ and $p$ are positive, relatively prime integers. The numbers $n_{i}$ for $i=1,2,3$ will always denote integers subject to the condition that

$$
r=\frac{1}{2}\left(\sum_{i} n_{i}-p-1\right)
$$

is a positive integer.

Let $X=S-\{0,1, \infty\}$ be the Riemann sphere minus three points. Consider the forms

$$
\omega_{n_{1} n_{2} n_{3}}=f\left(z_{1}, \ldots, z_{r}\right) \prod_{i=1}^{r} z_{i}^{\left(1-n_{3}\right) p^{\prime} / p}\left(1-z_{i}\right)^{\left(1-n_{2}\right) p^{\prime} / p} \prod_{1 \leqq i<j \leqq r}\left(z_{i}-z_{j}\right)^{2 p^{\prime} / p} d z_{1} \wedge \cdots \wedge d z_{r},
$$

with functions $f\left(z_{1}, \ldots, z_{r}\right)$ which are holomorphic on $X^{r}$, meromorphic on $S^{r}$ and symmetric under permutations of the $z_{i}$ 's. Let $\Delta\left(X^{r}\right)$ be the diagonal subspace $\bigcup_{i<j}\left\{z_{i}=z_{j}\right\}$ of $X^{r}$, and $X(r)=X^{r}-\Delta\left(X^{r}\right)$. The symmetric group $S(r)$ acts freely by permutations on $X(r)$, and the fundamental group $\pi_{1}(X(r) / S(r))$ of the braid space $X(r) / S(r)$ is a generalized braid group on $r$ strands.

$\omega_{n_{1} n_{2} n_{3}}$ is a complex, multivalued, $C^{\infty}$ holomorphic $r$-form on $X(r) / S(r)$, i.e. an element of the de Rham cohomology of $C^{\infty}$ differential forms on $X(r) / S(r)$ with values in a rank one local system $\mathscr{L}_{n_{1} n_{2} n_{3}}$ defined by its monodromy. Explicitly, let $\rho: \pi_{1}\left(X(r) / S(r), x_{0}\right) \rightarrow \mathbb{C}^{*}, \gamma \mapsto \rho(\gamma)$, be the representation of $\pi_{1}\left(X(r) / S(r), x_{0}\right)$ defined by the monodromy action $\gamma^{*} \omega_{n_{1} n_{2} n_{3}}\left(x_{0}\right)=\rho(\gamma) \cdot \omega_{n_{1} n_{2} n_{3}}\left(x_{0}\right)$ for any $x_{0} \in X(r) / S(r)$. Then we have, up to isomorphisms, a unique flat complex line bundle $\mathscr{L}_{n_{1} n_{2} n_{3}}$ on $X(r) / S(r)$ with characteristic homomorphism $\chi=\rho$. The elements $\gamma$ of $\pi_{1}\left(X(r) / S(r), x_{0}\right)$ act on a point $f_{0}$ of the fiber over $x_{0}$ moving it horizontally along $\gamma$ back to the point $\rho(\gamma) \cdot f_{0}$ over $x_{0}$. Thus, if $e\left(x_{0}\right)$ is a local section defined in a neighborhood of $x_{0}, \gamma$ acts as $\gamma e\left(x_{0}\right)=\rho(\gamma) \cdot e\left(x_{0}\right)$. 
The differential form $\omega$ has in general non-integrable singularities at the insertion points $0,1, \infty$ and vanishes on $\Delta\left(X^{r}\right)$. The natural support condition on the integration cycles $c$ is that the support of $c$, viewed as an $S(r)$-invariant subset of $X(r)$, be mapped by the inclusion $X(r) \rightarrow X^{r}$ to a set with compact closure. Denote by $H_{*}^{\varphi}\left(X(r) / S(r), \mathscr{L}_{n_{1} n_{2} n_{3}}\right)$ the homology with coefficients in $\mathscr{L}_{n_{1} n_{2} n_{3}}$ with this support condition.

Let $Y$ be the compact space obtained from the sphere $S$ by deleting three small open disks around the points 0,1 and $\infty$, and denote by $Y(r)$ the space $Y^{r}-\Delta\left(Y^{r}\right)$. Then, by a standard homotopy argument, we have the natural isomorphism

$$
H_{*}^{l f}\left(Y(r) / S(r), \mathscr{L}_{n_{1} n_{2} n_{3}}\right) \stackrel{\sim}{\rightarrow} H_{*}^{\varphi}\left(X(r) / S(r), \mathscr{L}_{n_{1} n_{2} n_{3}}\right),
$$

where $H_{*}^{l f}\left(Y(r) / S(r), \mathscr{L}_{n_{1} n_{2} n_{3}}\right)$ is the homology of the complex $C_{*}^{l f}\left(Y(r) / S(r), \mathscr{L}_{n_{1} n_{2} n_{3}}\right)$ of locally finite chains on $Y(r) / S(r)$ with coefficients in $\mathscr{L}_{n_{1} n_{2} n_{3}}$. This complex can be described as follows. Let $\tilde{Y}(r) / S(r)$ be the universal covering of $Y(r) / S(r)$, and $C_{*}^{l f}(\tilde{Y}(r)) \otimes \mathbb{C}$ the complex of locally finite chains (i.e. possibly infinite linear combinations of simplexes, such that only finitely many intersect any given compact set) on $\tilde{Y}(r)$ with complex coefficients. Then $C_{*}^{l f}\left(Y(r) / S(r), \mathscr{L}_{n_{1} n_{2} n_{3}}\right)=C_{*}^{l f}(\tilde{Y}(r)) \otimes_{\rho} \mathbb{C}$, where $C_{*}^{l f}(\tilde{Y}(r)) \otimes_{\rho} \mathbb{C}$ is the complex $C_{*}^{l f}(\tilde{Y}(r)) \otimes \mathbb{C}$ modulo the equivalence relation

$$
\tilde{c} \cdot \gamma \otimes \alpha \sim \tilde{c} \otimes \rho(\gamma) \alpha,
$$

for any $\tilde{c} \in C_{*}^{l f}(\tilde{Y}(r)), \alpha \in \mathbb{C}$ and $\gamma \in \pi_{1}\left(Y(r) / S(r), y_{0}\right)$ (acting on $\tilde{c}$ from the right).

We now compute the $r^{\text {th }}$ homology group relevant to the integration of $\omega_{n_{1} n_{2} n_{3}}$. The fundamental group $\pi_{1}(Y(r) / S(r))$ is a slight generalization of the braid group $B_{r}$ on $r$ strands, obtained by adding to the generators of $B_{r}$ the two elements representing loops around 0 and 1 . In other words: it is the subgroup of $B_{r+2}$ generated by the set $\left\{\sigma_{1}^{2}, \sigma_{i}, i=2, \ldots, r+1, \sigma_{r+2}^{2}\right\}$, where the standard generators $\sigma_{i}$ describe the positive simple braiding of the $i^{\text {th }}$ and $(i+1)^{\text {th }}$ strand. We have $\rho\left(\sigma_{1}^{2}\right)=q^{2\left(1-n_{3}\right)} ; \rho\left(\sigma_{i}\right)=-q^{2}, i=1, \ldots, r+1 ; \rho\left(\sigma_{r+2}^{2}\right)=q^{2\left(1-n_{2}\right)}$, where $q=e^{i \pi p^{\prime} / p}$.

Consider any subspace $A$ of $Y$ homotopic to the bouquet $A_{0}$ of two circles of radius $1 / 2$ centered at the points 0 and $1 . A_{0}$ is the subspace of the complex plane parametrized as the union

$$
A_{0}=\left\{z(\theta)=\frac{1}{2} e^{2 i \theta} \mid 0 \leqq \theta \leqq \pi\right\} \cup\left\{z(\theta)=\frac{1}{2}-\frac{1}{2} e^{2 i \theta} \mid \pi \leqq \theta \leqq 2 \pi\right\}
$$

of the two circles in their common point $\frac{1}{2}$. Let $A(r)=A^{r}-\Delta\left(A^{r}\right)$. Define

$$
\begin{aligned}
c_{j}=\left\{\left\{z_{1}\left(\theta_{1}\right), \ldots, z_{r}\left(\theta_{r}\right)\right\}\right. & \in A(r) / S(r) \mid 0 \leqq \theta_{1}, \ldots, \theta_{j} \leqq \pi, \\
& \left.\pi \leqq \theta_{j+1}, \ldots, \theta_{r} \leqq 2 \pi ; \theta_{1}<\cdots<\theta_{r}\right\}
\end{aligned}
$$

for $j=0, \ldots, r$. Also define $b_{l}$ to be the subset of $c_{l}$ defined by $\theta_{l+1}=0, l=0, \ldots, r-1$. The cells $c_{j}-b_{j}$ and $b_{l}$ give a cell decomposition of $A(r) / S(r)$. Our calculation is based on the assumption that the homomorphism induced by the inclusion $i: A \rightarrow Y$

$$
i_{*}: H_{*}^{l f}\left(A(r) / S(r), i^{*} \mathscr{L}\right) \rightarrow H_{*}^{l f}(Y(r) / S(r), \mathscr{L})
$$

is an isomorphism. This is clear for $r=1$, since $A$ is a proper deformation retract of $Y$, but appears technically difficult to prove in the general case. Thus what we actually compute is $H_{*}^{l f}\left(A(r) / S(r), i^{*} \mathscr{L}\right)$, and leave the above isomorphism as a 
conjecture. The boundary of $c_{j}$ is given by

$$
\partial c_{j}=\sum_{l=0}^{r-1} \partial_{l j} b_{l}
$$

where $\partial_{l j}$ is the $r \times(r+1)$ matrix

$$
\partial_{l j}=\left(q^{2\left(r-j-n_{2}\right)}-1\right) \delta_{l j}-\left(q^{2\left(n_{3}-j\right)}-1\right) \delta_{l+1, j} .
$$

Since there are no cells of dimension $r+1$, we have

$$
H_{r}^{l f}\left(Y(r) / S(r), \mathscr{L}_{n_{1} n_{2} n_{3}}\right)=\operatorname{Ker} \partial .
$$

The dimensions of the $r^{\text {th }}$ homology groups, considered as functions of the local system, are given by

Proposition. Assume that $q^{p}=1$ for some integer $p>1$ and $q^{j} \neq 1$ for $j=1, \ldots, p-1$. Given any integer $n$, let $\bar{n}$ be the unique integer in $\{0, \ldots, p-1\}$ such that $n=\bar{n}$ $(\bmod p)$. There are three cases

(1) $r \leqq \bar{n}_{2}-1$ and $r \leqq \bar{n}_{3}-1: \operatorname{dim} \operatorname{Ker} \partial=1$,

(2) $r \geqq \bar{n}_{2}$ and $\left(\overline{r-n_{2}}\right) \leqq \bar{n}_{3}-1: \operatorname{dim} \operatorname{Ker} \partial=2+\left(r-\bar{n}_{3}\right) \operatorname{div} p$,

(3) $r \geqq \bar{n}_{3}$ and $\left(r-n_{2}\right)>\bar{n}_{3}-1: \operatorname{dim} \operatorname{Ker} \partial=1+\left(r-\bar{n}_{3}\right) \operatorname{div} p$,

where $a \operatorname{div} b$ denotes the integer part of $a / b$ (i.e. the largest integer less than $a / b$ ).

To prove the proposition we notice that the elements of $\partial$ which are not identically zero lie on two diagonals. In case (1) none of the elements on the diagonals vanish. Thus $\operatorname{Ker} \partial=1$ because $\partial$ is a $r \times(r+1)$ matrix. In case (2) and (3) the diagonals have vanishing elements and $\partial$ decomposes into blocks of $i \times j$ matrices with $|i-j| \leqq 1$. The kernel of each block component is one-dimensional if $j=i+1$ and is empty otherwise. A simple analysis of the block decomposition of $\partial$ gives the result.

Note that case (1) of the above proposition could also be deduced as a corollary of a general result of Kohno [K], except that the support conditions are slightly different. If the hypothesis of case (1) is satisfied, Kohno's Theorem 1 implies that, in particular, $H_{*}^{l f}\left(Y(r) / S(r), \mathscr{L}_{n_{1} n_{2} n_{3}}\right)=H_{r}^{l f}\left(Y(r) / S(r), \mathscr{L}_{n_{1} n_{2} n_{3}}\right)$. The dimension of the only non-vanishing homology group is then equal to the absolute value of the Euler characteristic of $Y(r)$ divided by $r$ !.

3.4. Let us now consider the $r$-forms

$$
\omega_{m}=f\left(z_{1}, \ldots, z_{r}\right) \prod_{1 \leqq 1 \leqq r} z_{i}^{(1-m) p^{\prime} / p} \prod_{1 \leqq i<j \leqq r}\left(z_{i}-z_{j}\right)^{2 p^{\prime} / p} d z_{1} \wedge \cdots \wedge d z_{r},
$$

with functions $f\left(z_{1}, \ldots, z_{r}\right)$ holomorphic on $(S-\{0, \infty\})^{r}$, meromorphic on $S^{r}$ and symmetric in all arguments. This is a subcase of the one considered in Sect. 3.3. If $Z$ is the sphere minus two small open disks centered at 0 and $\infty$, the homology dual to the cohomology of the forms $\omega_{m}$ is $H_{r}^{l f}\left(Z(r) / S(r), \mathscr{L}_{m}\right)$, where $Z(r)=Z^{r}-\Delta\left(Z^{r}\right)$ and the local system $\mathscr{L}_{m}$ is the extension of $\mathscr{L}_{n_{1}, n_{2}=1, n_{3}}$, with $n_{3}=m$ and $n_{1}=p-n$ to $Z(r) / S(r)$. The calculation proceeds as in the previous paragraph. The cells $c_{j}$ are replaced by

$$
d_{r}=\left\{\left(z_{1}\left(\theta_{1}\right), \ldots, z_{r}\left(\theta_{r}\right)\right) \in T^{r} \mid 0 \leqq \theta_{1}<\cdots<\theta_{r} \leqq \pi\right\}
$$


where $T$ is a circle homotopic to $T_{0}=\left\{z(\theta)=\frac{1}{2} e^{2 i \theta} \mid 0 \leqq \theta \leqq \pi\right\}$ in $\mathbb{C}$. The boundary is $\partial d_{r}=\left(1-q^{2(r-m)}\right) b_{r-1}$, with $b_{r-1}$ the face of $d_{r}$ defined by $\theta_{r}=\pi$. Thus the $r^{\text {th }}$ homology group is one-dimensional and generated by $d_{r}$ if $r=m(\bmod p)$, and vanishes otherwise.

Note that for $r=m$ this is the cycle which defines the operator $Q_{m}$ in (2.4). Choosing the normalization as in Theorem 1, we have

$$
d(m)=\prod_{l=1}^{m} \frac{q^{2 l}-1}{q^{2}-1} d_{m} .
$$

3.5. The $N_{i}=\left(n_{i}, n_{i}^{\prime}\right)$ for $i=1,2,3$ will denote pairs in $\mathbb{Z}^{2}$ subject to the conditions that

$$
r=\frac{1}{2}\left(\Sigma n_{i}-p-1\right), \quad r^{\prime}=\frac{1}{2}\left(\Sigma n_{i}^{\prime}-p^{\prime}-1\right)
$$

are positive integers. Let us fix the points $O_{1}=\infty, O_{2}=1$ and $O_{3}=0$ on $S=\mathbb{C} \cup\{\infty\}$ in (3.1), (3.2). The coordinate is $z$ on $\mathbb{C}$ and $1 / z$ around the point at infinity. Other choices of coordinates are related to this one by conformal transformations. The forms $\omega_{N_{1} N_{2} N_{3}}\left(\infty, 1,0 ; \xi_{1}, \xi_{2}, \xi_{3}\right)$ introduced in (3.2) can be computed explicitly as

$$
\begin{aligned}
\omega_{N_{1} N_{2} N_{3}}= & f\left(z_{1}, \ldots, z_{r}, z_{1}^{\prime}, \ldots, z_{r^{\prime}}^{\prime}\right) \prod_{1 \leqq i \leqq r} \prod_{1 \leqq i^{\prime} \leqq r^{\prime}}\left(z_{i}-z_{i^{\prime}}^{\prime}\right)^{-2} \\
& \cdot \prod_{1 \leqq i \leqq r} z_{i}^{\left(1-n_{3}\right) p^{\prime} / p}\left(1-z_{i}\right)^{\left(1-n_{2}\right) p^{\prime} / p} \prod_{1 \leqq i<j \leqq r}\left(z_{i}-z_{j}\right)^{2 p^{\prime} / p} \\
& \cdot \prod_{1 \leqq i^{\prime} \leqq r^{\prime}} z_{i^{\prime}}^{\left(1-n_{3}^{\prime}\right) p / p^{\prime}}\left(1-z_{i^{\prime}}^{\prime}\right)^{\left(1-n_{2}^{\prime} p / p^{\prime}\right)} \prod_{1 \leqq i^{\prime}<j^{\prime} \leqq r^{\prime}}\left(z_{i^{\prime}}^{\prime}-z_{j^{\prime}}^{\prime}\right)^{2 p / p^{\prime}} d z_{1} \wedge \cdots \wedge d z_{r^{\prime}}^{\prime},
\end{aligned}
$$

with functions $f$ which are holomorphic on $X^{r} \times X^{r^{\prime}}$, meromorphic on $S^{r}$ and $S^{r^{\prime}}$, and symmetric in all their arguments. They are elements of the de Rham cohomology of $C^{\infty}$ differential forms on $X\left(r+r^{\prime}\right) / S(r) \times S\left(r^{\prime}\right)=\left[X(r) \times X\left(r^{\prime}\right)-\Delta\left(X(r) \times X\left(r^{\prime}\right)\right)\right] /$ $S(r) \times S\left(r^{\prime}\right)$ with values in the local system $\mathscr{L}_{N_{1} N_{2} N_{3}}$ on $X\left(r+r^{\prime}\right) / S(r) \times S\left(r^{\prime}\right)$ defined by the homomorphism $\tau: \pi_{1}\left(X\left(r+r^{\prime}\right) / S(r) \times S\left(r^{\prime}\right), x_{0}\right) \rightarrow \mathbb{C}^{*}$ associated with the monodromy of $\omega_{\mathrm{N}_{1} \mathrm{~N}_{2} \mathrm{~N}_{3}}$.

Note that the exponent of the factors $\left(z_{i}-z_{i}^{\prime}\right)$ in $\omega_{N_{1} N_{2} N_{3}}$ is an even integer for all $0 \leqq i \leqq r, 0 \leqq i^{\prime} \leqq r^{\prime}$. The subgroup of the braid group $\pi_{1}\left(X\left(r+r^{\prime}\right)\right)$ generated by the elements that represent the simple braiding of $z_{i}$ and $z_{i}^{\prime}$ for all $i$ and $i^{\prime}$ is in the kernel of $\tau$. Therefore, any local section of $\mathscr{L}_{N_{1} N_{2} N_{3}}$ defined in a neighborhood of a point close to the diagonal $\Delta\left(X(r) \times X\left(r^{\prime}\right)\right)$ can be continuously extended to a neighborhood of the diagonal in $X(r) \times X\left(r^{\prime}\right)$. We use the same notation $\mathscr{L}_{\mathrm{N}_{1} \mathrm{~N}_{2} \mathrm{~N}_{3}}$ for the local system so obtained on $X(r) / S(r) \times X\left(r^{\prime}\right) / S\left(r^{\prime}\right)$. The restrictions of $\mathscr{L}_{N_{1} N_{2} N_{3}}$ to $X(r) / S(r)$ and to $X\left(r^{\prime}\right) / S\left(r^{\prime}\right)$ are, respectively, $\mathscr{L}_{n_{1} n_{2} n_{3}}$ and $\mathscr{L}_{n_{1}^{\prime} n_{2}^{\prime} n_{3}^{\prime}}^{\prime}$ Therefore there is a monomorphism

$$
\begin{aligned}
& H_{r}^{l f}\left(X(r) / S(r), \mathscr{L}_{n_{1} n_{2} n_{3}}\right) \otimes H_{r^{\prime}}^{l f}\left(X\left(r^{\prime}\right) / S\left(r^{\prime}\right), \mathscr{L}_{n_{1}^{\prime} n_{2}^{\prime} n_{3}^{\prime}}^{\prime}\right) \\
& \rightarrow H_{r+r^{\prime}}^{l f}\left(X\left(r+r^{\prime}\right) / S(r) \times S\left(r^{\prime}\right), \mathscr{L}_{N_{1} N_{2} N_{3}}\right) .
\end{aligned}
$$

Thus the product of any $r$-cycle on $X(r)$ and any $r^{\prime}$-cycle on $X\left(r^{\prime}\right)$ gives an $\left(r+r^{\prime}\right)$ cycle on $X\left(r+r^{\prime}\right)$.

3.6. In this paragraph the data $M_{i}, M_{i}\left[j_{i}\right], r[j]$ and $r^{\prime}[j]$ are as in Sect. 3.2, with the condition that $r[j]$ and $r^{\prime}[j]$ be positive integers. 
Definition. We say that a triple $(L, M, N)$ of pairs in the Kac table $D$ obeys the fusion rules if $l=m+n-1(\bmod 2), l^{\prime}=m^{\prime}+n^{\prime}-1(\bmod 2)$ and

$$
\begin{gathered}
|m-n|+1 \leqq l \leqq \min (m+n-1,2 p-m-n-1), \\
\left|m^{\prime}-n^{\prime}\right|+1 \leqq l^{\prime} \leqq \min \left(m^{\prime}+n^{\prime}-1,2 p^{\prime}-m^{\prime}-n^{\prime}-1\right) .
\end{gathered}
$$

We say that the three-points vertex $\mathscr{V}_{M_{1} M_{2} M_{3}}$ obeys the fusion rules if the triple $\left(P-M_{1}, M_{2} M_{3}\right)$ does.

The three-point vertex, as it is defined in the following theorem, is given in general by an integral which is convergent but not absolutely convergent at the points in the integration domain where $z_{i}=z_{j}^{\prime}$ for some $i, j$. At these points, there is a violation of Ward identities. This problem does not arise if $p=2$ or $p^{\prime}=2$, since only $z$ variables or only $z^{\prime}$ variables are present in that case. We conjecture that there is a regularization prescription of the cycle of integration that solves the problem in the general case. More precisely, one should show that for each pair of cycles $c \otimes c^{\prime}$ in $H_{r}^{l f}\left(X(r) / S(r), \mathscr{L}_{n_{1} n_{2} n_{3}}\right) \otimes H_{r^{\prime}}^{l f}\left(X\left(r^{\prime}\right) / S\left(r^{\prime}\right), \mathscr{L}_{n_{1}^{\prime} n_{2}^{\prime} n_{3}^{\prime}}^{\prime}\right)$, there is a cycle representing an element of $H_{r+r^{\prime}}^{l f}\left(X\left(r+r^{\prime}\right) / S(r) \times S\left(r^{\prime}\right), \mathscr{L}_{N_{1} N_{2} N_{3}}\right)$, homologous to the image of $c \otimes c^{\prime}$, but with support in the complement of a tubular neighborhood of $\bigcup_{i j}\left\{z_{i}=z_{j}^{\prime}\right\}$. We have checked that such a cycle indeed exists in the case $r=r^{\prime}=1$, but a general proof is lacking.

Theorem 2. Suppose $p=2$ or $p^{\prime}=2$. Let $\mathscr{V}_{M_{1} M_{2} M_{3}}: \mathscr{F}_{M_{1}} \times \mathscr{F}_{M_{2}} \times \mathscr{F}_{M_{3}} \rightarrow \mathbb{C}$ be the degree zero trilinear form on Fock complexes $\mathscr{F}_{M_{i}}=\sum_{j_{1} \in \mathbb{Z}}^{M_{1}} \mathscr{F}_{M_{i}}^{\left(j_{i}\right)}$ defined in (3.1), with $c\left(M_{1}\left[j_{1}\right], M_{2}\left[j_{2}\right], M_{3}\left[j_{3}\right]\right)$ a cycle of $H_{r[j]}^{l f}\left(Y(r[j]) / S(r[j]), \mathscr{L}_{m_{1}\left[j_{1}\right] m_{2}\left[j_{2}\right] m_{3}\left[j_{3}\right]}\right) \otimes$ $H_{r^{\prime}[j]}^{l f}\left(Y\left(r^{\prime}[j]\right) / S\left(\left[r^{\prime}[j]\right]\right), \mathscr{L}_{m_{1}^{\prime}\left[j_{1}\right] m_{2}^{\prime}\left[j_{2}\right] m_{3}^{\prime}\left[j_{3}\right]}^{\prime}\right)$.

Then there exists a unique

$$
\mathscr{V}_{M_{1} M_{2} M_{3}}^{*}: H^{*}\left(\mathscr{F}_{M_{1}}\right) \times H^{*}\left(\mathscr{F}_{M_{2}}\right) \times H^{*}\left(\mathscr{F}_{M_{3}}\right) \rightarrow \mathbb{C}
$$

induced on cohomologies. $\mathscr{V}_{M_{1} M_{2} M_{3}}^{*}$ is nonvanishing if and only if it obeys the fusion rules.

Conjecture. Theorem 2 holds for any relatively prime positive integers $p, p^{\prime}$.

The conjecture is proven if there exists a regularization prescription for cycles containing both kinds of variables (see remark above). Note that the fusion rules for minimal models were obtained by Belavin, Polyakov and Zamolodchikov [BPZ] upon requiring that the "operator algebra" be closed. Theorem 2 gives, in particular, a topological derivation of the fusion rules.

Proof. It is organized in three steps.

(1) Assume that $\mathscr{V}_{M_{1} M_{2} M_{3}}$ obeys the fusion rules. These can be equivalently written as the inequalities $0 \leqq r[0] \leqq \min \left(m_{1}-1, m_{2}-1, m_{3}-1\right), 0 \leqq r^{\prime}[0] \leqq \min \left(m_{1}^{\prime}-1\right.$, $\left.m_{2}^{\prime}-1, m_{3}^{\prime}-1\right)$. Thus we have $0 \leqq r[j] \leqq \min \left(m_{1}\left[j_{1}\right]-1, m_{2}\left[j_{2}\right]-1, m_{3}\left[j_{3}\right]-1\right)$, $0 \leqq r^{\prime}[j] \leqq \min \left(m_{1}^{\prime}-1, m_{2}^{\prime}-1, m_{3}^{\prime}-1\right)$. From the Proposition in 3.3 , the homology groups $H_{r[j]}^{l f}\left(Y(r[j]) / S(r[j]), \mathscr{L}_{m_{1}\left[j_{1}\right] m_{2}\left[j_{2}\right] m_{3}\left[j_{3}\right]}\right)$ and $H_{r^{\prime}[j]}^{l f}\left(Y\left(r^{\prime}[j]\right) / S\left(r^{\prime}[j]\right.\right.$, $\left.\mathscr{L}_{m_{1}^{\prime}\left[j_{1}\right] m_{2}^{\prime}\left[j_{2}\right] m_{3}^{\prime}\left[j_{3}\right]}\right)$ are both one-dimensional. 


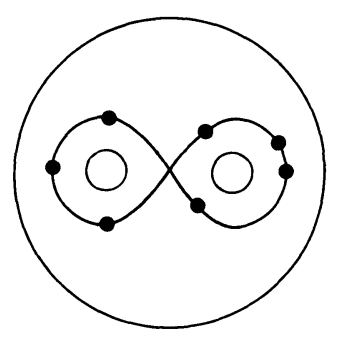

Fig. 1

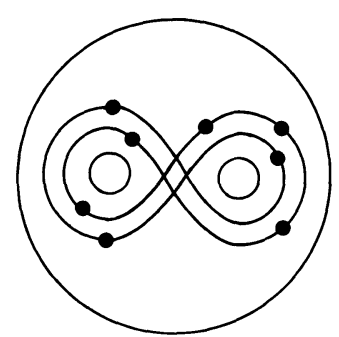

Fig. 2

The generating cycles are, respectively

$$
\begin{aligned}
& c\left(m_{1}\left[j_{1}\right], m_{2}\left[j_{2}\right], m_{3}\left[j_{3}\right]\right) \\
& =\sum_{k=0}^{r[j]}\left(\prod_{i=0}^{k-1} \frac{q^{2\left(r[j]-m_{2}\left[j_{2}\right]-i\right)}-1}{q^{2}-1} \prod_{i=k+1}^{r[j]} \frac{q^{2\left(m_{3}\left[j_{3}\right]-i\right)}-1}{q^{2}-1}\right) c_{k}, \\
& c^{\prime}\left(m_{1}^{\prime}\left[j_{1}\right], m_{2}^{\prime}\left[j_{2}\right], m_{3}^{\prime}\left[j_{3}\right]\right) \\
& =\sum_{k^{\prime}=0}^{r^{\prime}[j]}\left(\prod_{i=0}^{k^{\prime}-1} \frac{q^{\prime 2\left(r^{\prime}[j]-m_{2}^{\prime}\left[j_{2}\right]-i\right)}-1}{q^{\prime 2}-1} \prod_{i=k^{\prime}+1}^{r^{\prime}[j]} \frac{q^{\prime 2\left(m_{3}^{\prime}\left[j_{3}\right]-i\right)}-1}{q^{\prime 2}-1}\right) c_{k^{\prime}}^{\prime},
\end{aligned}
$$

where the chains $c_{k}$ are parametrized as in (3.5). In (3.8) $q^{\prime}=e^{i \pi p / p^{\prime}}$ and the chains $c_{\boldsymbol{k}^{\prime}}^{\prime}$ are

$c_{\boldsymbol{k}^{\prime}}^{\prime}=\left\{\left(z_{1}\left(\theta_{1}\right), \ldots, z_{\boldsymbol{r}^{\prime}}\left(\theta_{\boldsymbol{r}^{\prime}}\right)\right) \in A^{\prime \boldsymbol{r}^{\prime}} \mid 0 \leqq \theta_{1}, \ldots, \theta_{\boldsymbol{k}^{\prime}} \leqq \pi, \pi \leqq \theta_{\boldsymbol{k}^{\prime}+1}, \ldots, \theta_{\boldsymbol{r}^{\prime}} \leqq 2 \pi ; \theta_{1}<\cdots<\theta_{\boldsymbol{r}^{\prime}}\right\}$ for $k^{\prime}=1, \ldots, r^{\prime}-1$, where the bouquet $A^{\prime}$ is homotopic to the bouquet $A$ and chosen such that the intersection is as in Fig. 2. The cycle defining the three-points vertex is the product

$$
c_{M_{1}\left[j_{1}\right] M_{2}\left[j_{2}\right] M_{3}\left[j_{3}\right]}=\mathscr{N}_{M_{1}\left[j_{1}\right] M_{2}\left[j_{2}\right] M_{3}\left[j_{3}\right]} c_{m_{1}\left[j_{1}\right] m_{2}\left[j_{2}\right] m_{3}\left[j_{3}\right]} \otimes c_{m_{1}^{\prime}\left[j_{1}\right] m_{2}^{\prime}\left[j_{2}\right] m_{3}^{\prime}\left[j_{3}\right]}^{\prime}
$$

where $\mathscr{N}$ is an arbitrary normalization factor. $\mathscr{N}$ will be conveniently chosen (see Lemma 1).

The integral (3.1) can be explicitly computed [Fel 1; DoF] using techniques of contours deformation similar to those usually employed in integrals of the Pochammer-Selberg type (see e.g. [WW], [HK] and [TK]). One then verifies that 
it is not identically zero. We conclude that the three-point vertex $\mathscr{V}_{M_{1} M_{2} M_{3}}$ is uniquely defined and nonvanishing if it obeys the fusion rules.

(2) Let $Q_{(i)}$ for $i=1,2,3$ be the coboundary operators acting on the tensor product of three complexes $\mathscr{F}_{M_{i}}$ as $Q_{(1)}=Q \otimes 1 \otimes 1, Q_{(2)}=1 \otimes Q \otimes 1$ and $Q_{(3)}=1 \otimes 1 \otimes Q$. The composites $\mathscr{V}_{M_{1} M_{2} M_{3}} \cdot Q_{(i)}: \mathscr{F}_{M_{1}} \times \mathscr{F}_{M_{2}} \times \mathscr{F}_{M_{3}} \rightarrow \mathbb{C}$ are trilinear forms of degree one. Their components on $\mathscr{F}_{\boldsymbol{M}_{1}}^{\left(j_{1}\right)} \times \mathscr{F}_{\boldsymbol{M}_{2}}^{\left(j_{2}\right)} \times \mathscr{F}_{\boldsymbol{M}_{3}}^{\left(j_{3}\right)}$ are $\mathscr{V}_{\boldsymbol{M}_{1}\left[j_{1}+1\right] M_{2}\left[j_{2}\right] M_{3}\left[j_{3}\right]}\left(Q^{\left(j_{1}\right)} \xi_{1}, \xi_{2}, \xi_{3}\right)$, $\mathscr{V}_{M_{1}\left[j_{1}\right] M_{2}\left[j_{2}+1\right] M_{3}\left[j_{3}\right]}\left(\xi_{1}, Q^{\left(j_{2}\right)} \xi_{2}, \xi_{3}\right)$ and $\mathscr{V}_{M_{1}\left[j_{1}\right] M_{2}\left[j_{2}\right] M_{3}\left[j_{3}+1\right]}\left(\xi_{1}, \xi_{2}, Q^{\left(j_{3}\right)} \xi_{3}\right)$ with $\sum_{i} j_{i}+1=0$.

Using (3.1), (3.2) and the definition of the operator $Q$ given in Theorem 1, we can express them as the integrals $\int_{\Gamma_{(i)}} \omega_{P+M_{1}\left[j_{1}\right], M_{2}\left[j_{2}\right], M_{3}\left[j_{3}\right]}$ of the same $\left(r+r^{\prime}\right)$-form $\left(r=\frac{1}{2}\left(\sum_{i} m_{i}\left[j_{i}\right]-1\right), r^{\prime}=\frac{1}{2}\left(\sum_{i} m_{i}^{\prime}-p^{\prime}-1\right)\right.$; see 3.5$)$ over the three cycles $\Gamma_{(i)}$ schematically represented in Fig. 3.

The $\Gamma_{(i)}$ are given by the products of a cycle $c_{(i)}$ defining the respective threepoint vertex and a cycle $d_{(i)}$ supported on a circle around $\infty, 1$ or 0 defining $Q_{(1)}, Q_{(2)}$ or $Q_{(3)}$. They are elements of $\left.H \otimes H^{\prime}=H_{r}^{l f}(Y(r) / S(r)), \mathscr{L}_{p+m_{1}\left[j_{1}\right], m_{2}\left[j_{2}\right], m_{3}\left[j_{3}\right]}\right) \otimes$ $H_{r^{\prime}}^{l f}\left(Y\left(r^{\prime}\right) / S\left(r^{\prime}\right), \mathscr{L}_{m_{1}^{\prime}, m_{2}^{\prime}, m_{3}^{\prime}}^{\prime}\right)$.

Let us assume that $\mathscr{V}_{M_{1} M_{2} M_{3}}$ obeys the fusion rules. Then the numbers $r$ and $r^{\prime}$ satisfy the inequalities $m_{2}\left[j_{2}\right] \leqq r, r-m_{2}\left[j_{2}\right] \leqq m_{3}\left[j_{3}\right]-1$ and $0 \leqq r^{\prime} \leqq$ $\min \left(m_{1}^{\prime}-1, m_{2}^{\prime}-1, m_{3}^{\prime}-1\right)$.

From the Proposition in 3.3, we see that $\operatorname{dim} H=2$ and $\operatorname{dim} H^{\prime}=1$. Therefore the $\Gamma_{(i)}$ are linear combinations of only two inequivalent cycles. On the other hand, an explicit verification shows that they are in fact pairwise independent. It follows that there is precisely one linear relation between the three composites $\mathscr{V}_{M_{1} M_{2} M_{3}} \cdot Q_{(i)}$.

We have proven the first part of the

Lemma 1. Assume that $\mathscr{V}_{M_{1} M_{2} M_{3}}$ obeys the fusion rules. Then

(i) (BRST invariance). There are nonvanishing complex coefficients $\lambda_{i}$ for which $\sum_{i=1}^{3} \lambda_{i} \mathscr{V}_{M_{1} M_{2} M_{3}} \cdot Q_{(i)}=0$

(ii) Fix the normalization of the cycle (3.9) defining $\mathscr{V}_{M_{1} M_{2} M_{3}}$ to be

$$
\begin{array}{rlrl}
\mathscr{N}_{M_{1}\left[j_{1}\right] M_{2}\left[j_{2}\right] M_{3}\left[j_{3}\right]} & =\varepsilon\left[j_{2}, j_{3}\right], \text { for } j_{2}, j_{3} \text { even } & \\
& =\varepsilon\left[j_{2}, j_{3}\right](-1)^{\left(m_{3}-1\right)\left(m_{2}^{\prime}-1\right)} q^{-\left(m_{1}+m_{2} m_{3}\right)} & & \text { for } j_{2} \text { odd, } j_{3} \text { even } \\
& =\varepsilon\left[j_{2}, j_{3}\right](-1)^{\left(p-m_{1}\right) m_{2}^{\prime}+m_{3}} q^{-\left(m_{1}+m_{2} m_{3}\right)} & & \text { for } j_{2} \text { even, } j_{3} \text { odd } \\
& =\varepsilon\left[j_{2}, j_{3}\right](-1)^{p\left(m_{2}^{\prime}-1\right)+p^{\prime}\left(m_{2}-1\right)+m_{2} m_{2}^{\prime}} & & \text { for } j_{2}, j_{3} \text { odd },
\end{array}
$$
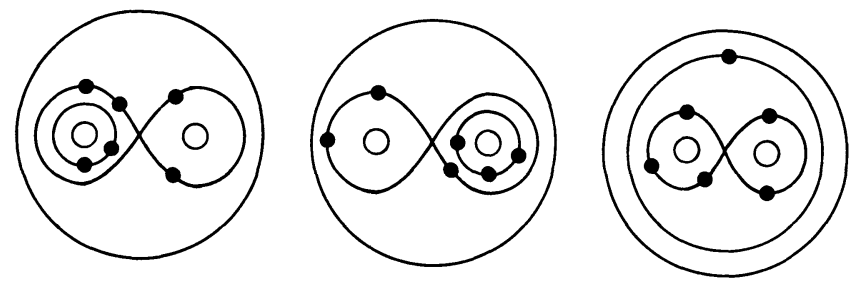

Fig. 3. 
where $\varepsilon\left[j_{2}, j_{3}\right]=(-1)^{k_{2}\left[p\left(m_{3}^{\prime}-1\right)-p^{\prime}\left(m_{3}-1\right)\right]+k_{3}\left[p\left(m_{2}^{\prime}-1\right)-p^{\prime}\left(m_{2}-1\right)\right]}$, and $k_{i}=\frac{1}{2} j_{i}$ for $j_{i}$ even, $k_{i}=\frac{1}{2}\left(j_{i}-1\right)$ for $j_{i}$ odd. Let $J_{i}$ be the grading operators which take the values $j_{i}$ on $\mathscr{F}_{M_{i}}^{\left(j_{i}\right)}$. We have

$$
\mathscr{V}_{M_{1} M_{2} M_{3}} \cdot Q_{(1)}=\mathscr{V}_{M_{1} M_{2} M_{3}} \cdot Q_{(2)}+\mathscr{V}_{M_{1} M_{2} M_{3}} \cdot(-1)^{J_{2}} \cdot Q_{(3)}
$$

Part (ii) of the lemma is the commutation property of the three-point operators (obtained from the vertex by duality) and the coboundary $Q$ (see 4.1). The proof relies on the explicit form of the cycles defining the three terms of the above equation.

As an immediate corollary of point (i) of the lemma: $\mathscr{V}_{M_{1} M_{2} M_{3}}$ is well defined on the product of cohomologies if it obeys the fusion rules. For the restriction $\overline{\mathscr{V}}_{M_{1} M_{2} M_{3}}$, defined by $\overline{\mathscr{V}}_{M_{1}\left[j_{1}\right] M_{2}\left[j_{2}\right] M_{3}\left[j_{3}\right]}: \operatorname{Ker} Q^{\left(j_{1}\right)} \times \operatorname{Ker} Q^{\left(j_{2}\right)} \times \operatorname{Ker} Q^{\left(j_{3}\right)} \rightarrow \mathbb{C}$, vanishes on all submodules $\operatorname{Im} Q^{\left(j_{i}-1\right)} \subset \operatorname{Ker} Q^{\left(j_{i}\right)}$.

(3) It remains to prove that the restriction $\overline{\mathscr{V}}_{M_{1} M_{2} M_{3}}$ vanishes if it does not obey the fusion rules. Let us consider the case where the fusion rules are violated by the first components $m_{1}\left[j_{1}\right], m_{2}\left[j_{2}\right]$ and $m_{3}\left[j_{3}\right]$ of the pairs $M_{1}\left[j_{1}\right], M_{2}\left[j_{2}\right]$ and $M_{3}\left[j_{3}\right]$. Then the above vanishing statement follows from the fact that $\mathscr{V}_{M_{1} M_{2} M_{3}}$ can be written as a linear combination of the composites $\mathscr{V}_{M_{1} M_{2} M_{3}} \cdot Q_{(i)}$.

As an example, consider the case where $0 \leqq r[j] \leqq \min \left(m_{1}\left[j_{1}\right]-1, m_{2}\left[j_{2}\right]-1\right)$, $r[j] \geqq m_{3}\left[j_{3}\right], 0 \leqq r^{\prime}[j] \leqq \min \left(m_{1}^{\prime}-1, m_{2}^{\prime}-1, m_{3}^{\prime}-1\right)$. Let $c \otimes c^{\prime}$ be the $\left(r[j]+r^{\prime}[j]\right)-$ product cycle defining the three-points vertex. From the Proposition in 3.3, we have that the classes of $c$ and $c^{\prime}$ are both unique. $c^{\prime}$ is given by (3.8). $c$ can be computed as the kernel of the boundary matrix in 3.3 in the form of a linear combination of the chains $c_{0}, c_{1}, \ldots, c_{r[j]-m_{3}\left[j_{3}\right]}$ in (3.4). Let $d\left(m_{3}\left[j_{3}\right]\right)$ be the $m_{3}\left[j_{3}\right]$-cycle (3.6) defining the BRST operator $Q$ acting on $\mathscr{F}_{M_{3}}^{\left(j_{3}\right)}$. The product of $c_{m_{1}\left[j_{1}\right], m_{2}\left[j_{2}\right], p-m_{3}\left[j_{3}\right]}$ in $(3.7)$ and $d\left(m_{3}\left[j_{3}\right]\right)$ gives a cycle of $H_{r[j]}^{l f}(Y(r[j]) / S(r[j])$, $\left.\mathscr{L}_{m_{1}\left[j_{1}\right], m_{2}\left[j_{2}\right], m_{3}\left[j_{3}\right]}\right)$ which is necessarily homologous to $c$. We have thus proved that $\mathscr{V}_{M_{1} M_{2} M_{3}}=\mathscr{V}_{M_{1} M_{2} M_{3}} \cdot Q_{(3)}$.

More general violations of the fusion rules can be analyzed in a similar way. The cases where these are violated by the second components of the pairs $M_{1}, M_{2}$ and $M_{3}$ do not require any new proof. The argument lies in the fact that, if we exchange $\beta_{+}$with $\beta_{-}$(i.e. $p$ with $p^{\prime}$ ), a complex isomorphic to that of Theorem 1 can be defined with a coboundary $Q^{\prime}$ constructed in terms of $V_{\beta}$ - screening operators. A violation of the fusion rules implies that $\mathscr{V}_{M_{1} M_{2} M_{3}}$ be equal to a linear combination of composites $\mathscr{V}_{M_{1} M_{2} M_{3}} \cdot Q_{(i)}^{\prime}$. The proof of the theorem is now complete.

Observe that, in the notation of $3.1, \mathscr{V}_{M_{1} M_{2} M_{3}}^{*},\left(\mathscr{V}^{*} \cdot \psi_{1}^{*} \cdot \psi_{2}^{*}\right)_{M_{1} M_{2} M_{3}}$, $\left(\mathscr{V}^{*} \cdot \psi_{1}^{*} \cdot \psi_{3}^{*}\right)_{M_{1} M_{2} M_{3}}$ and $\left(\mathscr{V}^{*} \cdot \psi_{2}^{*} \cdot \psi_{3}^{*}\right)_{M_{1} M_{2} M_{3}}$ satisfy the same fusion rules. It can be easily verified that they are in fact proportional. The same observation applies to $\left(\mathscr{V}^{*} \cdot \psi_{1}^{*}\right)_{M_{1} M_{2} M_{3}},\left(\mathscr{V}^{*} \cdot \psi_{2}^{*}\right)_{M_{1} M_{2} M_{3}},\left(\mathscr{V}^{*} \cdot \psi_{3}^{*}\right)_{M_{1} M_{2} M_{3}}$ and $\left(\mathscr{V}^{*} \cdot \psi_{1}^{*} \cdot \psi_{2}^{*} \cdot \psi_{3}^{*}\right)_{M_{1} M_{2} M_{3}}$. They are nonvanishing and all proportional if and only if $\left(M_{1}, M_{2}, M_{3}\right)$ obeys the fusion rules. In view of the remarks of Sect. 3.1, this fact implies that there are several equivalent representations of minimal models' three-points conformal blocks $C_{M_{1} M_{2} M_{3}}$. They are expressed as integrals of multivalued forms of maximal degree on braid spaces of different dimensions. For example, $C_{M_{1} M_{2} M_{3}}$ can be identified with $\mathscr{V}_{M_{1} M_{2} M_{3}}^{*}$ when this is nonzero and with $\left(\mathscr{V}^{*} \cdot \psi_{3}^{*}\right)_{M_{1} M_{2} M_{3}}$ otherwise. An explicit form for the isomorphisms $\psi_{i}^{*}$ ("background operators") is given in 4.2. 


\section{Conformal Blocks on Genus $g$}

4.1. We will formulate "sewing" [G; So; MoS] using operators rather than trilinear formis. The difference is merely in notation; however, this choice makes the notion of summing over "intermediate states" somewhat more direct. The dependence of three-points vertices and operators on the points will be usually left as implicit when no ambiguity can arise.

Three-points operators are the operators obtained from the three-points vertex by duality. For example $\mathscr{V}_{\boldsymbol{M}_{2} \boldsymbol{M}_{3}}^{\boldsymbol{M}_{1}}: \mathscr{F}_{\boldsymbol{M}_{2}} \otimes_{\mathbb{C}} \mathscr{F}_{\boldsymbol{M}_{3}} \rightarrow \mathscr{F}_{M_{1}}$ is the three-points operator

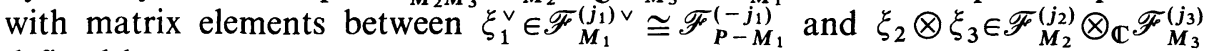
defined by

$$
\left\langle\xi_{1}^{\vee}, \mathscr{V}_{\boldsymbol{M}_{2}\left[j_{2}\right] M_{3}\left[j_{3}\right]}^{\boldsymbol{M}_{1}\left[j_{2}\right]} \xi_{2} \otimes \xi_{3}\right\rangle=\mathscr{V}_{\left(P-M_{1}\right)\left[-j_{1}\right] M_{2}\left[j_{2}\right] M_{3}\left[j_{3}\right]}\left(\xi_{1}^{\vee}, \xi_{2}, \xi_{3}\right),
$$

with $j_{1}=j_{2}+j_{3}$. It is expressed as the integral

$$
\int_{\boldsymbol{M}_{2}\left[j_{12}\right] M_{3}\left[j_{3}\right]}^{M_{1}\left[j_{2}\left[j_{2}\right] M_{3}\left[j_{3}\right]\right.}
$$

of the multivalued operator obtained from (3.2) by duality on the cycle $c_{M_{2}\left[j_{2}\right] M_{3}\left[j_{3}\right]}^{\left.\left.M_{1}\right] j_{1}\right]}=$ $c_{\left(P-M_{1}\right)\left[-j_{1}\right] M_{2}\left[j_{2}\right] M_{3}\left[j_{3}\right]^{*}}$

Analogously, we define $\mathscr{V}_{M_{3}}^{M_{1} M_{2}}: \mathscr{F}_{M_{3}} \rightarrow \mathscr{F}_{M_{1}} \otimes_{\mathbb{C}} \mathscr{F}_{M_{2}}, \mathscr{V}_{M_{1} M_{2} M_{3}}: \mathscr{F}_{M_{1}} \otimes_{\mathbb{C}} \mathscr{F}_{M_{3}} \rightarrow$ $\mathscr{F}_{M_{2}}, \ldots$ As a rule, the direction of the mapping goes from the lower to the upper indices, and (the matrix elements of) an operator with upper indices $M_{k}$ are defined by the corresponding three-points vertex with indices $P-M_{k}$.

The operators with indices $M_{1}, M_{2}, M_{3}$, one of which is upper and two lower, obey the fusion rules if the triple $\left(M_{1}, M_{2}, M_{3}\right)$ does. Those with two upper indices and one lower obey the fusion rules if $\left(P-M_{1}, M_{2}, M_{3}\right)$ does.

Lemma 1 and Theorem 2 have obvious versions for three-point operators. In particular, point (ii) of Lemma 1 implies that, with the normalization there established:

$$
Q \mathscr{V}_{M_{2} M_{3}}^{M_{1}}=\mathscr{V}_{M_{2} M_{3}}^{M_{1}} Q \otimes 1+\mathscr{V}_{M_{2} M_{3}}^{M_{1}}(-1)^{J} \otimes Q
$$

Theorem 1 ensures that $\mathscr{V}_{M_{2} M_{3}}^{* M_{1}}: H^{*}\left(\mathscr{F}_{M_{2}}\right) \otimes_{\mathbb{C}} H^{*}\left(\mathscr{F}_{M_{3}}\right) \rightarrow H^{*}\left(\mathscr{F}_{M_{1}}\right)$ is uniquely defined and nonvanishing if and only if it obeys the fusion rules. Analogous statements apply to $\mathscr{V}_{M_{3}}^{* M_{1} M_{2}}, \mathscr{V}_{M_{1} M_{3}}^{* M_{2}}, \ldots$

4.2. Observe that the Fock space $\mathscr{F}_{P-1}^{(0)}=F\left(2 \beta_{0}, \beta_{0}\right)$ has vanishing highest weight. Consider the degree zero bilinear form $\mathscr{B}_{\mathbf{M}_{1} \mathbf{M}_{2}}\left(\mathrm{O}_{1}, \mathrm{O}_{2}, Q\right): \mathscr{F}_{M_{1}} \times \mathscr{F}_{M_{2}} \rightarrow \mathbb{C}$ with components given by

$$
\mathscr{B}_{M_{1}[-j] M_{2}[j]}\left(O_{1}, O_{2}, Q ; \xi_{1}, \xi_{2}\right)=\mathscr{V}_{M_{1}[-j] P-1 M_{2}[j]}\left(O_{1}, Q, O_{2} ; \xi_{1}, v, \xi_{2}\right),
$$

where $v$ is the highest weight vector of $\mathscr{F}_{P-1}^{(0)}$. Since $h_{P-1}=0, \mathscr{B}_{M_{1} M_{2}}\left(O_{1}, O_{2}, Q\right)$ does not depend on the reference point $Q \in S$ where we insert the primary vertex operator with charge $\beta_{P-1}=2 \beta_{0}$. The dual two-points operator $\mathscr{B}_{M_{2}}^{M_{1}}\left(O_{1}, O_{2}, Q\right)$ ("background operator") induces $\mathscr{B}_{M_{2}}^{* M_{1}}\left(O_{1}, O_{2} ; Q\right): H^{*}\left(\mathscr{F}_{M_{2}}\right) \rightarrow H^{*}\left(\mathscr{F}_{M_{1}}\right)$ on cohomology which is nonvanishing if and only if it obeys the fusion rule $M_{1}=P-M_{2}$.

Let $O_{1}=\infty$ and $O_{2}=0$ on $S=\hat{\mathbb{C}} \cdot \psi_{M_{2}}^{* M_{1}}$ denotes $\mathscr{B}_{M_{2}}^{* M_{1}}(\infty, 0 ; Q)$ with the choice of coordinates $z$ on $\widehat{\mathbb{C}}$ and $1 / z$ around infinity. It can be easily shown that $\psi_{M}^{* P-M}$ commutes with $L_{k}$ for $k \in \mathbb{Z}$ and thus defines a canonical module isomorphism 
mapping every vector of $H^{*}\left(\mathscr{F}_{M}\right)$ to its dual in $H^{*}\left(\mathscr{F}_{P-M}\right)$. The composites $\psi_{P-M_{1}}^{* M_{1}} \cdot \mathscr{V}_{M_{2} M_{3}}^{* P-M_{1}}, \mathscr{V}_{P-M_{2} M_{3}}^{* M_{1}} \cdot \psi_{M_{2}}^{* P-M_{2}}$ and $\mathscr{V}_{M_{2} P-M_{3}}^{* M_{1}} \cdot \psi_{M_{3}}^{* P-M_{3}}$ are proportional.

From the concluding remarks of 3.6, a representation for minimal models' three-point operators $C_{M_{2} M_{3}}^{M_{1}}$ is given by $\mathscr{V}_{M_{2} M_{3}}^{*_{M_{1}}}$ if $\left(M_{1}, M_{2}, M_{3}\right)$ obeys the fusion rules and by $\mathscr{V}_{M_{2} P-M_{3}}^{* M_{1}} \cdot \psi_{M_{3}}^{* P}-M_{3}$ if $\left(M_{1}, M_{2}, P-M_{3}\right)$ obeys the fusion rules. Let $\mu_{i}$ be $M_{i}$ or $P-M_{i}$. We then conclude that $C_{M_{2} M_{3}}^{M_{1}}$ and the duals $C_{M_{1} M_{2} M_{3}}, C_{M_{3}}^{M_{1} M_{2}}, \ldots$ are all nonvanishing if and only if one of the triples $\left(\mu_{1}, \mu_{2}, \mu_{3}\right)$ obeys the fusion rules.

4.3. Let $X$ be a three-punctured Riemann sphere $S \backslash\{a, b, c\}$ together with a choice of local analytic coordinates $z_{(a)}, z_{(b)}$ and $z_{(c)}$ which vanish at, respectively, $a, b$ and $c . X$ will be denoted by the graphical symbol of a vertex with three incident edges, where each edge (or "leg") corresponds to a puncture. To $X$ we associate threepoint operators $\mathscr{V}^{*}(a, b, c)$ defined on cohomology with representation indices $L, M$ and $N$ corresponding to the vertex operators inserted respectively at the points $a, b$ and $c$. A three-point operator is represented by an analogous graphical symbol, where now each leg corresponds to a cohomology module and carries a representation index and an arrow. The orientation of the arrows is an agreement with the direction of the mapping between cohomology modules. In Fig. 4 the symbols representing (i) $\mathscr{V}_{M N}^{* L}(a, b, c): H^{*}\left(\mathscr{F}_{M}\right) \otimes_{\mathbb{C}} H^{*}\left(\mathscr{F}_{N}\right) \rightarrow H^{*}\left(\mathscr{F}_{L}\right)$ and (ii) $\mathscr{V}_{N}^{* L M}(a, b, c): H^{*}\left(\mathscr{F}_{N}\right) \rightarrow H^{*}\left(\mathscr{F}_{L}\right) \otimes_{\mathbb{C}} H^{*}\left(\mathscr{F}_{M}\right)$ are given as examples.

The generic expression "sewing" will be understood as a sequence of elementary sewings. An elementary sewing is a surgery operation of three-punctured Riemann spheres. It proceeds in two steps. Let $p$ and $q$ be two punctures on either the same or two distinct spheres, $z_{(p)}$ and $z_{(q)}$ be local coordinates defined around them. In the first step we delete two small open disks centered at $p$ and $q$ on the sphere (or the spheres) to which $p$ and $q$ belong. Secondly, we identify, on annuli around $p$ and $q$, the local coordinates by setting $z_{(p)}=z_{(q)}^{-1}$. In the general case, moduli parameters are introduced in the definition of the local coordinates. We say that sewing has been performed "around $p$ and $q$."

Graphically, we represent an elementary sewing operation by joining with a solid line two legs of either the same or of two distinct three-punctured spheres.

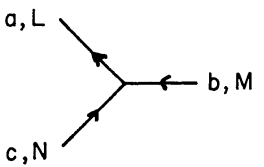

(i)

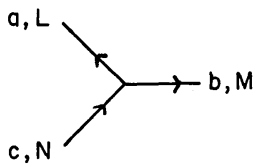

(ii)

Fig. 4

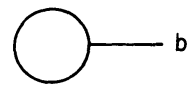

(i)

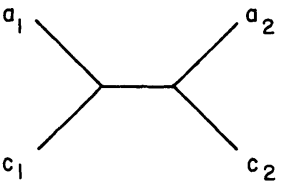

(ii)

Fig. 5 
The respective sewing diagrams are drawn in Fig. 5: (i) is the one-punctured torus obtained by sewing $X$ around its punctures $a$ and $c$; (ii) is the four-punctured sphere obtained by sewing $X_{1}=S_{1}-\left\{a_{1}, b_{1}, c_{1}\right\}$ and $X_{2}=S_{2}-\left\{a_{2}, b_{2}, c_{2}\right\}$ around the punctures $b_{1}$ and $b_{2}$.

The associated elementary sewing of three-point operators has an analogous graphical representation, where two legs can be joined if they carry equal representation indices and have compatible orientations. It is defined by the following operations: (1) sew around the punctures associated with the two legs; (2) if $L$ is the common representation index of the two legs, take a sum over all "intermediate states", i.e. over a basis $\xi_{j}$ of vectors of definite weight of $H^{*}\left(\mathscr{F}_{L}\right)$.

Concretely, diagram 6(i) represents the map from $H^{*}\left(\mathscr{F}_{M}\right) \ni \eta$ to $\mathbb{C}$ defined by

$$
\sum_{j}\left\langle\xi_{j}^{\vee}, \mathscr{V}_{M L}^{* L} \eta \otimes \xi_{j}\right\rangle
$$

associated with the one-punctured torus 5(i). Here, $\xi_{j}^{\vee}$ denotes the dual basis with $\left\langle\xi_{j}^{\vee}, \xi_{l}\right\rangle=\delta_{j l}$. This expression depends on the puncture $b$ and on a modular parameter introduced in the sewing operation. This dependence is prescribed by the coordinate dependence of three-point operators. Diagram 6(ii) represents the four-point operator mapping $H^{*}\left(\mathscr{F}_{N_{1}}\right) \otimes_{\mathbb{C}} H^{*}\left(\mathscr{F}_{N_{2}}\right) \ni \xi_{1} \otimes \xi_{2}$ to $H^{*}\left(\mathscr{F}_{L_{1}}\right) \otimes H^{*}\left(\mathscr{F}_{L_{2}}\right)$ whose matrix elements are

$$
\sum_{j}\left\langle\omega_{1}, \mathscr{V}_{M N_{1}}^{* L_{1}} \eta_{j} \otimes \xi_{1}\right\rangle\left\langle\omega_{2} \otimes \eta_{j}^{\vee}, \mathscr{V}_{N_{2}}^{* L_{2} M} \xi_{2}\right\rangle
$$

where $\omega_{i}$ are in the dual spaces. It is associated with the four-punctured sphere in 5(ii).

Note that a concrete computation of the above sums over cohomology modules is made possible by replacing them with suitable sums over complexes. From property (4.1), the sum over $H^{*}\left(\mathscr{F}_{L}\right)=H^{0}\left(\mathscr{F}_{L}\right)$ in (4.2) is equal to the alternated sum

$$
\sum_{i \in \mathbb{Z}}(-1)^{i} \sum_{j}\left\langle\xi_{j}^{(i) \vee}, \mathscr{V}_{M[0] L[i]}^{L[i]} \eta \otimes \xi_{j}^{(i)}\right\rangle
$$

over the complex $\mathscr{F}_{L}$. Here $\xi_{j}^{(i)}$ is a basis of $\mathscr{F}_{L}^{(i)}$, and $\xi_{j}^{(i) \vee}$ the dual basis in the dual complex. On the other hand, the sum in (4.3) could be obviously replaced by a sum where $\eta$ ranges over a basis of the entire complex $\mathscr{F}_{M}$. For, in virtue of (4.1), all terms with $\eta$ not in the cohomology are identically zero.

Sums over Fock spaces can be computed using coherent state techniques [DiPFHLS; Fel 1].

L

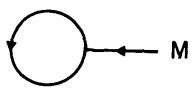

(i)

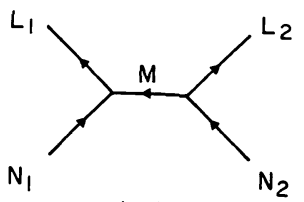

(ii)

Fig. 6 


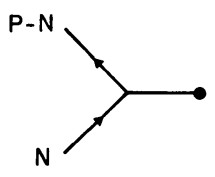

Fig. 7

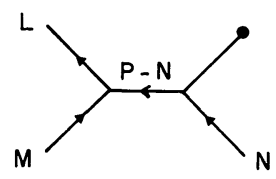

Fig. 8

In order to complete the set of graphical rules, we also need a symbol for the background operators $B_{N}^{* P-N}(a, b ; Q)$. This is represented in Fig. 7. The dot corresponds to the reference point $Q$.

The sewing of a three-point operator $\mathscr{V}_{M P-N}^{* L}\left(a, b, d_{1}\right)$ and a background operator $B_{N}^{* P-N}\left(c, d_{2} ; Q\right)$ is drawn in Fig. 8. The resulting three-point operator is associated with $X=S-\{a, b, c\}$ and coincides with $\mathscr{V}_{M P-N}^{* L}(a, b, c) \cdot \psi_{N}^{* P-N}$, of which Fig. 8 establishes the graphical symbol.

Finally, the construction of conformal blocks of minimal models on a Riemann surface is formulated as follows. A $k$-punctured surface $\Sigma_{g, k}$ of genus $g$ can be obtained by sewing a number $(2 g-2+k)$ of three-punctured spheres. The same $\Sigma_{g, k}$ may be represented by different sewing diagrams, according to differently chosen patterns of elementary sewings. These diagrams are related by transformations of the mapping class group of $\Sigma_{g, k}$. To every sewing diagram representing $\Sigma_{g, k}$ we associate the $k$-point operator obtained by sewing three-point operators $\mathscr{V}^{*}$ (Fig. 4) or $\mathscr{V}^{*} \cdot \psi^{*}$ (Fig. 8) according to the same diagram. The matrix elements of this operator are minimal models' $k$-points conformal blocks on genus $g$. In particular, the matrix element taken between $k$ highest weight vectors is a primary conformal block.

4.4. We call genus $g$ characters the zero-point conformal blocks on genus $g$ defined by sewing. For $g=1$, they are the characters $\chi_{L}(q)=\operatorname{Tr}_{H^{*}\left(\mathscr{F}_{L}\right)} q^{L_{0}}$ of $L\left(h_{L}, c\right) \cong H^{*}\left(\mathscr{F}_{L}\right)$, where $q=\exp (2 \pi i \tau)$ with $\operatorname{Im} \tau>0 . \chi_{L}(q)$ is computed by sewing a single operator $\mathscr{V}_{1 L}^{* L}(\infty, 1,0)$ with the choice $z_{(\infty)}=\frac{1}{u}$ and $z_{(0)}=q u$ of local coordinates around $\infty$ and 0 . On higher genus, let us choose a specific sewing diagram for $\Sigma_{g}$ in the form of two one-punctured tori and $(g-2)$ two-punctured tori connected by their legs. Fix the canonical basis for $H_{1}\left(\Sigma_{g}, \mathbb{Z}\right)$ which is given by the $a_{i}$ and $b_{i}$ cycles of the $g$ tori, and let $\tau$ be the period matrix on the Siegel upper half-plane defined in terms of a basis of abelian differential $v_{i}$ of first kind as $\int_{a_{i}} v_{j}=\delta_{i j}, \int_{b_{i}} v_{j}=\tau_{i j}$. The genus $g$ characters $\chi$ corresponding to the sewing diagram for $\Sigma_{g}$ are labelled by the sets $\{L, M, N\}$ of representation indices $L_{1}, \ldots, L_{g}, M_{1}, \ldots, M_{g-1}, N_{2}, \ldots, N_{g-1}$ and turn out to be functions of $\tau$. The characters corresponding to any other 


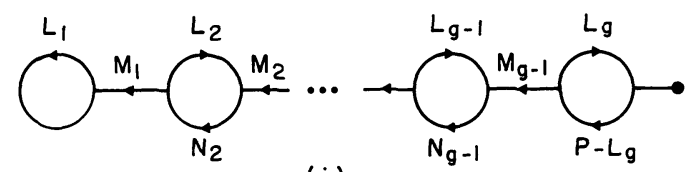

(i)

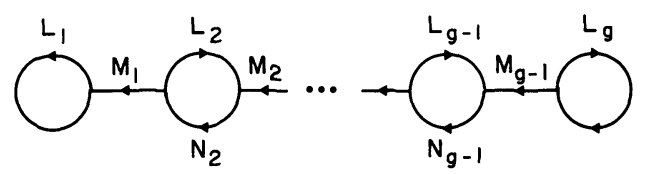

(ii)

Fig. 9

choice of sewing diagram can be obtained from the above ones via the action of the symplectic group $S p(2 g, \mathbb{Z})$ on $\tau$. The action of $S p(2 g, \mathbb{Z})$ on the genus $g$ characters is calculable, in principle, from the results in [Fel S2].

$\chi\{L, M, N\}$ is represented in diagram 9(i) for $g$ even and in 9(ii) for $g$ odd. A parity argument based on the fusion rules shows that a number $s$ of three-point operators (with $s-g=1 \bmod 2$ ) must have the representation of Fig. 8. We choose the smallest numbers $s=1$ for $g$ even and $s=0$ for $g$ odd. All other possibilities give in fact proportional results.

The traces over the cohomology modules $H^{*}\left(\mathscr{F}_{L_{i}}\right)$ are written as alternated sums over the respective complexes, as in (4.4). Using then (4.1), we easily see that all sums over intermediate vector states in $H^{*}\left(\mathscr{F}_{M_{i}}\right)$ and $H^{*}\left(\mathscr{F}_{N_{i}}\right)$ can be equivalently replaced by sums over the larger Fock spaces $\mathscr{F}_{M_{i}}^{(0)}$ and $\mathscr{F}_{N_{i}}^{\left(j_{i}\right)}$, for all additional terms so introduced are equal to zero. Thus

$$
\chi\{L, M, N\}=\sum_{j_{1}, \ldots, j_{g} \in \mathbb{Z}^{g}}\left(\prod_{i=1}^{g}(-1)^{j_{i}}\right) K\{L[j], M, N[j]\},
$$

where

$$
\begin{aligned}
& K\{L[j], M, N[j]\} \\
& =\sum_{\xi_{1} \in \mathscr{F}_{L_{1}}^{\left(j_{1}\right)}} \cdots \sum_{\xi_{g} \in \mathscr{F}_{L_{g}}^{\left(j_{g}\right)}} \sum_{\eta_{1} \in \mathscr{F}_{M_{g-1}}^{(0)}} \cdots \sum_{\eta_{g-1} \in \mathscr{F}_{M_{g-1}}^{(0)}} \sum_{\zeta_{2} \in \mathscr{F}_{N_{2}}^{\left(j_{2}\right)}} \cdots \sum_{\zeta_{g-1} \in \mathscr{F}_{N_{g-1}}^{\left(j_{g}-1\right)}} \\
& \cdot\left\langle\xi_{1}^{\vee}, \mathscr{V}_{M_{1} N_{1}\left[j_{1}\right]}^{L_{1}\left[j_{1}\right]} \eta_{1} \otimes \xi_{1}\right\rangle\left\langle\xi_{2}^{v} \otimes \eta_{1}^{v} \mathscr{V}_{N_{2}\left[j_{2}\right]}^{L_{2}\left[j_{2}\right] M_{1}} \zeta_{2}\right\rangle\left\langle\zeta_{2}^{v}, \mathscr{V}_{M_{2} L_{2}\left[j_{2}\right]}^{N_{2}\left[j_{2}\right]} \eta_{2} \otimes \xi_{2}\right\rangle \cdots
\end{aligned}
$$

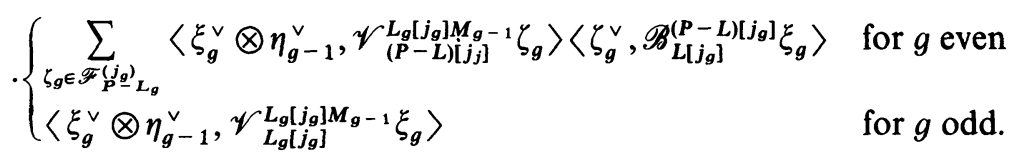

The notation $\sum_{\xi \in \mathscr{F}}$ means the sum over a basis of vectors with definite weight, and $\xi^{\vee}$ denotes the element of the dual basis dual to the basis element $\xi$.

Each three-point operator is expressed as an integral over a cycle supported on the associated three-punctured sphere. $K\{L[j], M, N[j]\}$ has then the form 


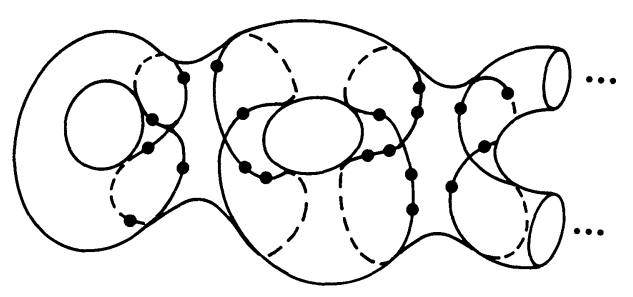

Fig. 10

of an integral

$$
\int_{\Gamma\{L[j], M, N[j]\}} \Omega_{\{L[j], M, N[j]\}}
$$

over the cycle given by the product of $c_{M_{1} N_{1}\left[j_{1}\right]}^{L_{1}\left[j_{1}\right]}, c_{N_{2}\left[j_{2}\right]}^{L_{2}\left[j_{2}\right]}, c_{M_{2} L_{2}\left[j_{2}\right]}^{N_{2}\left[j_{2}\right]} \ldots$. This cycle is drawn in Fig. 10. Each of its factors is given by (3.9) as a product cycle times a normalization. Let $\delta_{i}=(-1)^{j_{i}}$ be the parity of $j_{i}$. Using the fact that every three-point operator in diagram 9 obeys the fusion rules, one can show that $\Gamma\{L[j], M, N[j]\}$ depends only on the parity $\delta=\left(\delta_{1}, \ldots, \delta_{g}\right)$ of $j_{1}, \ldots, j_{g}$. For an integer $n$ such that $0 \leqq n \leqq p-1$, let $n[\delta]$ be the integer equal to $n$ if $\delta=1$ and equal to $(p-n)$ if $\delta=-1$. As a result:

$$
\Gamma\{L[j], M, N[j]\}=\Gamma_{\delta}\{L, M, N\}
$$

is given by the product of

$$
\begin{aligned}
& c_{l_{1}\left[-\delta_{1}\right], m_{1}, l_{1}\left[\delta_{1}\right]} \otimes c_{p^{\prime}-l_{1}^{\prime}, m_{1}^{\prime}, l_{1}^{\prime}}^{\prime}, c_{l_{2}\left[-\delta_{2}\right], p-m_{1}, n_{2}\left[\delta_{2}\right]} \otimes c_{p^{\prime}-l_{2}^{\prime}, p^{\prime}-m_{1}^{\prime}, n_{2}^{\prime}}^{\prime}, \\
& c_{n_{2}\left[-\delta_{2}\right], m_{2}, l_{2}\left[\delta_{2}\right]} \otimes c_{p-n_{2}^{\prime}, m_{2}^{\prime}, n_{2}^{\prime}}^{\prime}, \ldots,
\end{aligned}
$$

each cycle being supported as in Fig. 10, times the normalization factor

$$
\begin{aligned}
\mathscr{N}_{\delta}\{L, M, N\}= & \prod_{i=1}^{g} q^{1 / 2\left(1-\delta_{i}\right) l_{i}\left(1-m_{i}\right)} \prod_{i=2}^{g-1} q^{1 / 2\left(1-\delta_{i}\right) n_{i}\left(m_{i}-1+1\right)} \\
& \cdot q^{2 l g} \text { for } g \text { even } 1 \text { for } g \text { odd. }
\end{aligned}
$$

The integrand in (4.6) can be computed explicitly. The direct method [DiPFHLS] makes use of a concrete (Schottky) parametrization of $\Sigma_{g}$. The sums over Fock spaces are evaluated and shown to be convergent. A simpler method is suggested by the observation that the integrand in (4.6) is a conformal block of a conformal field theory constructed upon: a pair $\mathscr{A}, \bar{A}$ of commuting Heisenberg algebras; a representation space $\bigoplus_{N \in \mathbb{Z}^{2}} F\left(\beta_{N}, \beta_{0}\right) \otimes_{\mathbb{C}} \bar{F}\left(\beta_{N}, \beta_{0}\right)$; the duality product introduced in Sect. 2. This theory has a formulation in terms of a single scalar field $\phi: \Sigma_{g} \rightarrow \mathbb{R} / 2 \pi \beta_{+} \mathbb{Z}$, with action $[\mathrm{Fr}]$

$$
S(\phi)=\frac{1}{8 \pi} \int d^{2} x \sqrt{\gamma}\left(\gamma^{\mu \alpha} \partial_{\mu} \phi \partial_{\alpha} \phi+2 i \beta_{0} R \phi\right)
$$

where $R$ is the scalar curvature of a metric $\gamma$ given on $\Sigma_{g}$. Local correlation 
functions are path-integral expectation values of products $\prod \exp \left(i \beta_{a} \phi\left(P_{a}\right)\right)$ obeying the neutrality condition $\sum_{a} \beta_{a}=-2 \beta_{0}(g-1)$. The path-integral is computed by a saddle-point method. Let us choose a complex coordinate system for which $\gamma$ takes the local form $d s^{2}=\rho(z, \bar{z})|d z|^{2}$. The analytic structure of local correlation functions was analyzed by E. Verlinde and H. Verlinde [VV1].

The same analysis can be repeated in our case. We first observe that $\Omega_{\{L[j], M, N[j]\}}$ is a conformal block of the expectation value

$$
\begin{aligned}
& \left\langle\prod_{i=1}^{(1 / 2) g(p-2)} e^{i \beta+\phi\left(P_{i}\right)} \prod_{i=1}^{(1 / 2) g\left(p^{\prime}-2\right)} e^{i \beta-\phi\left(P_{i^{\prime}}^{\prime}\right)} e^{i 2 \beta_{0} \phi\left(Q_{0}\right)}\right\rangle \text { for } g \text { even, } \\
& \left\langle\prod_{i=1}^{(1 / 2)(g-1)(p-2)} e^{i \beta+\phi\left(P_{i}\right)} \prod_{i=2}^{(1 / 2)(g-1)\left(p^{\prime}-2\right)} e^{i \beta-\phi\left(P_{i}^{\prime}\right)}\right\rangle \text { for } g \text { odd. }
\end{aligned}
$$

Here the total number of screening operators is computed by summing the numbers of those associated with each three-point operator of diagrams 9(i) and 9(ii). A diagrammatic algorithm can be easily derived, which shows that the total numbers of $\beta_{+}$and $\beta_{-}$screening operators are, respectively, equal to the topological numbers $\frac{1}{2}\{p$ (\# of arrows) $-(p+1)$ (\# of vertices) -2 (\# of dots) $\}$ and $\frac{1}{2}\left\{p^{\prime}\right.$ (\# of arrows) $\left(p^{\prime}+1\right)$ (\# of vertices) $-2(\#$ dots $\left.)\right\}$. This makes it clear why they depend only on the genus and not on the representation labels $\{L[j], M, N[j]\}$.

Secondly, we compute the path-integral. Let the canonical basis $a_{i}, b_{i}$ chosen as specified above, $v_{i}$ be the corresponding abelian differentials of first kind with $\tau$ as period matrix. We use the following standard notations. Given on $\Sigma_{g}$ two positive divisors $A$ and $B$ having the same degree, $\int_{A}^{B} v$ with $v=\left(v_{1}, \ldots, v_{g}\right)$ is the Jacobi map to $J\left(\Sigma_{g}\right)=\mathbb{C}^{g} /\left(\mathbb{Z}^{g}+\tau \mathbb{Z}^{g}\right) . E(P, Q)$ is the accordingly defined prime form. Finally, let $\Delta$ be the Riemann divisor class and $\int_{(g-1) P}^{\Delta} v$ be the projection to $J\left(\Sigma_{g}\right)$ of the vector of Riemann constants with base-point $P$. By $\sigma(P)$ we denote the unique homomorphic. $g / 2$-differential having: no zeroes, no poles, and multipliers 1 and $\exp \left\{i \pi(g-1) \tau_{j j}-2 \pi i \int_{(g-1) P}^{\Delta} v_{j}\right\}$ when $P$ is moved around, respectively, $a_{j}$ and $b_{j} . \sigma(P)$ was introduced by Fay [F, p. 31] (see also [VV1]).

Let us fix the metric $d s^{2}=\rho|d z|^{2}$. Then [VV1]

$$
\left\langle\prod_{a=1}^{k} \exp \left(i \beta_{a} \phi\left(P_{a}\right)\right)\right\rangle=\left[e^{c S_{L}(\rho)} \prod_{a} \rho\left(P_{a}\right)^{-k\left(\beta_{a}\right)}\right] \sum_{L_{1}, \ldots, L_{g} \in \mathbb{Z}^{2}}\left|s_{\{L\}}\left(P_{1}, \ldots, P_{k}\right)\right|^{2}
$$

where $S_{L}(\rho)=\frac{i}{48 \pi} \int(\partial \log \rho d z)^{\wedge} \wedge(\bar{\partial} \log \rho d \bar{z})$ is the Liouville action. The conformal blocks $s_{\{L\}}=s_{\left(L_{1}, \ldots, L_{g}\right)}$ are sections of a line bundle over the $k$-fold product $J\left(\Sigma_{g}\right) \times \cdots \times J\left(\Sigma_{g}\right)$ minus its diagonal subspace. Explicitly:

$$
\begin{aligned}
s_{\{L\}}\left(P_{1}, \ldots, P_{k}\right)= & \left(\operatorname{det}^{\prime} \partial\right)^{-1 / 2} \exp \left\{i \pi \beta_{\{L\}} \cdot \tau \cdot \beta_{\{L\}}+i 2 \pi \beta_{\{L\}} \cdot\left[\sum_{a} \beta_{a} \int_{P_{0}}^{P_{a}} v+2 \beta_{0} \int_{(g-1) P_{0}}^{\Delta} v\right]\right\} \\
& \cdot \prod_{a} \sigma\left(P_{a}\right)^{-2 \beta_{0} \beta_{a}} \prod_{a<b} E\left(P_{a}, P_{b}\right)^{\beta_{a} \beta_{b}},
\end{aligned}
$$


where $\operatorname{det}^{\prime} \partial$ is the holomorphic square root of the regularized determinant of the Laplacian without zero mode and $\beta_{\{L\}}=\left(\beta_{L_{1}}, \ldots, \beta_{L_{g}}\right)$. Note that, in virtue of the condition $\sum_{a} \beta_{a}=-2 \beta_{0}(g-1)$, the above expression does not depend on the basepoint $P_{0}$. Putting $\{L\}$ equal to $\{L[j]\}$, some of the charges $\beta_{a}$ equal to $\beta_{+}$, some equal to $\beta_{-}$and one equal to $2 \beta_{0}$ if $g$ is even, we obtain an explicit formula for $\Omega_{\{L[j] M N[j]\}}$. Note that this formula depends only on $\{L[j]\}$.

The final part of the computation is straightforward. We have $\sqrt{2 p p^{\prime}} \beta_{L_{i}\left[j_{i}\right]}=$ $l_{i}^{\prime} p-l_{i} p^{\prime}-\left(p-p^{\prime}\right)+j_{i} p p^{\prime}$ if $j_{i}$ is even and $\sqrt{2 p p^{\prime}} \beta_{L_{i}\left[j_{i}\right]}=l_{i}^{\prime} p+l_{i} p^{\prime}-\left(p-p^{\prime}\right)+$ $\left(j_{i}-1\right) p p^{\prime}$ if $j_{i}$ is odd. Set

$$
\begin{aligned}
\Lambda[\delta] & =\left(\lambda_{1}\left[\delta_{1}\right], \ldots, \lambda_{g}\left[\delta_{g}\right]\right), & \lambda_{i}\left[\delta_{i}\right] & =l_{i}^{\prime} p-\delta_{i} l_{i} p^{\prime}, \\
\varepsilon & =\left(\varepsilon_{1}, \ldots, \varepsilon_{g}\right), & \varepsilon_{i} & =p-p^{\prime} .
\end{aligned}
$$

We perform the sums in (4.5): over $k_{i}\left[\delta_{i}=1\right]=\frac{1}{2} j_{i} \in \mathbb{Z}$ for $j_{i}$ even and over $k_{i}\left[\delta_{i}=-1\right]=\frac{1}{2}\left(j_{i}-1\right)$ for $j_{i}$ odd. The result is

$$
\chi\{L, M, N\}=\sum_{\delta_{1}, \ldots, \delta_{g}= \pm 1}\left(\prod_{i=1}^{g} \delta_{i}\right) \int_{\Gamma_{\delta}\{L, M, N\}} \Omega_{\delta}\{L\},
$$

where, if $g$ is even

$$
\begin{aligned}
\Omega_{\delta}\{L\}= & \Delta_{\Lambda[\delta]}\left(Z_{\mathrm{even}} \mid \tau\right) \prod_{\substack{i, j=1 \\
i<j}}^{g(p-2) / 2} E\left(P_{i}, P_{j}\right)^{2 p^{\prime} / p} \\
& \cdot \prod_{\substack{i^{\prime}, j^{\prime}=1 \\
i^{\prime}<j^{\prime}}}^{g\left(p^{\prime}-2\right) / 2} E\left(P_{i^{\prime}}^{\prime}, P_{j^{\prime}}^{\prime}\right)^{2 p / p^{\prime}} \prod_{i=1}^{g(p-2) / 2} \prod_{i^{\prime}=1}^{g\left(p^{\prime}-2\right) / 2} E\left(P_{i}, P_{i^{\prime}}^{\prime}\right)^{-2} \\
& \cdot \prod_{i=1}^{g(p-2) / 2} \sigma\left(P_{i}\right)^{2-2 p^{\prime} / p} \prod_{i^{\prime}=1}^{g\left(p^{\prime}-2\right) / 2} \sigma\left(P_{i^{\prime}}^{\prime}\right)^{2-2 p / p^{\prime}} \sigma(Q)^{-4+2 p^{\prime} / p+2 p / p^{\prime}} \\
& \cdot \prod_{i=1}^{g(p-2) / 2} E\left(P_{i}, Q\right)^{-2+2 p^{\prime} / p} \prod_{i^{\prime}=1}^{g\left(p^{\prime}-2\right) / 2} E\left(P_{i^{\prime}}^{\prime}, Q\right)^{-2+2 p / p^{\prime}} \\
& \cdot d z\left(P_{1}\right) \wedge \cdots \wedge d z\left(P_{1}^{\prime}\right) \wedge \cdots ;
\end{aligned}
$$

if $g$ is odd

$$
\begin{aligned}
& \Omega_{\delta}\{L\}=\Delta_{\Lambda[\delta]}\left(Z_{\text {odd }} \mid \tau\right) \prod_{\substack{i, j=1 \\
i<j}}^{(g-1)(p-2) / 2} E\left(P_{i}, P_{j}\right)^{2 p^{\prime} / p} \prod_{\substack{i^{\prime}, j^{\prime}=1 \\
i^{\prime}<j^{\prime}}}^{(g-1)\left(p^{\prime}-2\right) / 2} E\left(P_{i^{\prime}}^{\prime}, P_{j^{\prime}}^{\prime}\right)^{2 p / p^{\prime}} \\
& \cdot \prod_{i=1}^{(g-1)(p-2) / 2} \prod_{i=1}^{(g-1)\left(p^{\prime}-2\right) / 2} E\left(P_{i}, P_{i^{\prime}}^{\prime}\right)^{-2} \prod_{i=1}^{(g-1)(p-2) / 2} \sigma\left(P_{i}\right)^{2-2 p^{\prime} / p} \\
& (g-1)\left(p^{\prime}-2\right) / 2 \\
& \prod_{i^{\prime}=1} \sigma\left(P_{i^{\prime}}^{\prime}\right)^{2-2 p / p^{\prime}} d z\left(P_{1}\right) \wedge \cdots \wedge d z\left(P_{1}^{\prime}\right) \wedge \cdots ;
\end{aligned}
$$

and

$$
\begin{aligned}
\Delta_{\Lambda}(Z \mid \tau)= & \sum_{k \in \mathbb{Z} g} \exp \left\{i \pi\left(2 p p^{\prime} k+\Lambda-\varepsilon\right) \tau\left(2 p p^{\prime} k+\Lambda-\varepsilon\right)^{t} / 2 p p^{\prime}\right. \\
& \left.+i 4 \pi\left(2 p p^{\prime} k+\Lambda-\varepsilon\right) Z^{t} / 2 p p^{\prime}\right\}
\end{aligned}
$$




$$
\begin{aligned}
Z_{\text {even }}= & \sum_{i=1}^{g(p-2) / 2} p^{\prime} \int_{P_{0}}^{P_{i}} v-\sum_{i^{\prime}=1}^{g\left(p^{\prime}-2\right) / 2} p \int_{P_{0}}^{P_{i^{\prime}}^{\prime}} v-\left(p-p^{\prime}\right)\left[\int_{P_{0}}^{Q} v+\int_{(g-1) P_{0}}^{\Delta} v\right], \\
Z_{\text {odd }}= & \sum_{i=1}^{(g-1)(p-2) / 2} p^{\prime} \int_{P_{0}}^{P_{i}} v-\sum_{i^{\prime}=1}^{\Delta} \int_{P_{0}}^{(g-1)\left(p^{\prime}-2\right) / 2} v-\left(p-p^{\prime}\right) \int_{(g-1) P_{0}}^{\Delta} v .
\end{aligned}
$$

Acknowledgements. G. F. thanks P. Orlik for correspondence, J. Kramer for discussions, and the Institute for theoretical physics at UCSB, for hospitality. R. S. thanks P. Deligne and A. Dimca for suggestions, as well as ETH, where this work was completed, for hospitality. The main part of this work was completed while the second author was at the Institute for Advanced Study, supported by the NSF under grant DMS- 8610730 .

\section{References}

[FeiFu] Feigin, B. L., Fuchs, D. B.: Representations of the Virasoro algebra. In: Seminar on supermanifolds. Leites, D. (ed.), Reports of the Dept. Math. Stockholm Univ. (1986)

[BPZ] Belavin, A. A., Polyakov, A. M., Zamolodchikov, A. B.: Infinite conformal symmetry in two-dimensional quantum field theory. Nucl. Phys. B241, 333-380 (1984)

[Fel 1] Felder, G.: BRST approach to minimal models. Nucl. Phys. B317, 215-236 (1989)

[FelS 1] Felder, G., Silvotti, R.: Free field representation of minimal models on a Riemann surface. Phys. Lett. B231, 411-416 (1989)

[Fel 2] Felder, G.: Minimal models on a Riemann surface. In: Recent developments in conformal field theories. Randjbar-Daemi, S., Zuber, J.-B. (eds.). Singapore: World Scientific 1990

[FelS 2] Felder, G., Silvotti, R.: Modular covariance of minimal model correlation functions. Commun. Math. Phys. 123, 1-15 (1989)

[TK] Tsuchyia, A., Kanie, Y.: Fock space representations of the Virasoro algebra Intertwining operators. Publ. RIMS 22, 259-327 (1986)

[K] Kohno, T.: Homology of a local system on the complement of hyperplanes. Proc. Jpn Acad. 62, Ser. A, 144-147 (1986)

[DoF] Dotsenko, Vl. S., Fateev, V. A.: Conformal algebra and multipoint correlation functions in 2d statistical models. Nucl. Phys. B240 [FS12], 312-348 (1984); Fourpoint correlation functions and operator algebra in $2 \mathrm{~d}$ conformal invariant theories with central charge $\leqq 1$. Nucl. Phys. B251 [FS13], 691-734 (1985); Operator algebra of two-dimensional conformal theories with central charge $C \leqq 1$. Phys. Lett. 154B, 291-295 (1985)

[WW] Whittaker, E., Watson, G.: A course of modern analysis. Cambridge: Cambridge University Press 1940

[HK] Hattori, A., Kimura, T.: On the Euler integral representation of hypergeometric functions in several variables. J. Math. Soc. Jpn 26, 1 (1974)

[DiPFHLS] Di Vecchia, P., Pezzella, F., Frau, M., Hornfeck, K., Lerda, A., Sciuto, S.: N-point $g$-loop vertex for a free bosonic theory with vacuum charge. Nucl. Phys. B322 (1989)

[Fr] Friedan, D.: In: Unified String Theory. Green, M., Gross, D. (eds.). Singapore: World Scientific 1986

[VV1] Verlinde, E., Verlinde, H.: Chiral bosonisation, determinants and the string partition function. Nucl. Phys. B288, 357-396 (1987)

[F] Fay, J. D.: Theta functions on Riemann surfaces. Berlin, Heidelberg, New York: Springer 1973

[DeM] Deligne, P., Mostow, M.: Monodromy of hypergeometric functions and non-lattice integral monodromy. Publ. Math. IHES 63, 5 (1986)

[So] Sonoda, H.: Sewing conformal field theories I. Nucl. Phys. B311, 401-416 (1988/89); Sewing conformal field theories II. Nucl. Phys. B311, 417-432 (1988/89)

[MoS] Moore, G., Seiberg, N.: Classical and quantum conformal field theory. Commun. Math. Phys. 123, 177-254 (1989) 
[G] Gawędzki, K.: Wess-Zumino-Witten Conformal Field Theory, IHES/P/89/06 preprint, Lectures given at the Summer School Constructive Quantum Field Theory II, Erice 1988

[A] Aomoto, K.: Gauss-Manin connection of integrals of difference products. J. Math. Soc. Jpn 39, 191-208 (1987)

[DaJMM] Date, E., Jimbo, M., Matsuo, A., Miwa, T.: Hypergeometric type integrals and the $s l(2, \mathbb{C})$ Knizhnik-Zamolodchikov equation, preprint RIMS-667 (1989)

[Sc V] Schechtman, V. V., Varchenko, A. N.: Integral representation of $N$-point conformal correlators in the WZW model, MPI Bonn/89-51 (1989)

[Fei S V] Feigin, B. L., Schechtman, V. V., Varchenko, A. N.: On algebraic equations satisfied by correlators in Wess-Zumino-Witten models. Lett. Math. Phys. 20, 291-297 (1990)

[Ma] Matsuo, A.: An application of Aomoto-Gelfand hypergeometric functions to the $S U(n)$ Knizhnik-Zamolodchikov equation. Commun. Math. Phys. 134, 65-77 (1990)

[L] Lawrence, R. J.: Homological representations of the Hecke algebra. Commun. Math. Phys. 135, 141-191 (1990)

Communicated by A. Jaffe 